Check for updates

Cite this: Mater. Adv., 2021, 2, 146

Received 11th September 2020 Accepted 26th November 2020

DOI: 10.1039/d0ma00697a

rsc.li/materials-advances

\section{Solution-based "bottom-up" synthesis of group VI transition metal dichalcogenides and their applications}

\begin{abstract}
Áine Coogan (D) and Yurii K. Gun'ko (D) *
Research interest in the area of transition metal dichalcogenide (TMD) nanostructures has significantly increased in recent years. This interest is driven by their exceptional properties (e.g. semiconductor state, electronic confinement, conductivity, flexibility, transparency, large surface area, etc.) and their potential applications as materials for catalysis, photonics, sensing, solid lubrication, energy storage, high performance electronics, imaging, medical diagnostics and drug delivery systems, to name a few. The most common methods of production of these nanomaterials are "top-down" techniques involving solution-phase exfoliation or mechanical microcleavage, or chemical vapour deposition, a "bottom-up" approach. However, these methods suffer from a number of drawbacks, including poor controllability over size, high cost, and lack of scalability, thus limiting their potential commercial use. Therefore, over the last number of years, solution-based "bottom-up" synthesis of TMDs is gaining much attention as it can provide solutions to these problems. This review focuses on "bottom-up" methods such as hydro/ solvothermal, hot-injection and other approaches which have the potential to produce high-quality TMD nanostructures with controllable properties such as size, phase, morphology, and at low-cost. Here, we consider and compare various "bottom-up" synthetic techniques for synthesis of TMD nanostructures, explore some potential applications of corresponding colloidal TMD materials and provide future outlook for this fastdeveloping and important area.
\end{abstract}

\section{Introduction}

Transition metal dichalcogenides (TMDs) have been researched as far back as the 1960s. In recent years, however, the area has experienced a huge surge in interest. Research in the area of $2 \mathrm{D}$ materials in general has soared of late, and can be traced back to 2004, when graphene was first isolated from graphite by Novoselov et al. by the method of mechanical exfoliation. ${ }^{1}$ While this method produced high-quality samples of graphene, it was not scalable, thus limiting large-scale applications of the material. In 2008, Coleman et al. reported liquid-phase exfoliation (LPE) of graphite, which opened the door for mass production of graphene through a facile, top-down approach. ${ }^{2}$ This approach was later expanded upon by the same group, and was found to be effective in the exfoliation of a range of 2D materials, such as boron nitride (BN) and TMDs, among others. ${ }^{3}$

The interest in TMDs of late can also be attributed to their versatility and a range of exciting properties. For example, they have variable electrical properties: depending on their structure, they can be metallic or semiconducting. ${ }^{4,5}$ They exhibit a

School of Chemistry, CRANN and AMBER Research Centers, Trinity College Dublin, College Green, Dublin 2, Ireland. E-mail: igounko@tcd.ie tunable bandgap which is dependent on the number of monolayers, and an indirect to direct bandgap transition on being reduced from the bulk to the monolayer. ${ }^{5}$ Semiconducting monolayer TMDs such as $\mathrm{MoS}_{2}$ and $\mathrm{WS}_{2}$ also exhibit photoluminescence in the visible region of the spectrum. ${ }^{6}$ These properties make them potential candidates for use in optoelectronics and sensing. ${ }^{7,8}$

Although the method of LPE partially addressed the issue of scalability, it presented other problems. The best results from LPE are often obtained using high-boiling point organic solvents, such as $N$-methyl-2-pyrrolidone (NMP) and dimethylformamide (DMF), which are toxic and hard to remove from the end product. ${ }^{9}$ Bodik et al. also showed that exfoliation of TMDs, specifically $\mathrm{MoS}_{2}$, at high starting concentrations in more 'green' media such as ethanol-water mixtures can lead to the formation of unwanted side-products. ${ }^{10}$ LPE also has poor controllability over the number of layers and lateral sizes of nanosheets formed, often requiring further post-processing steps to achieve monodisperse samples. ${ }^{11}$ Thus, LPE-produced 2D nanosheet dispersions may not be of suitable monodispersity or quality for many applications, particularly in the case of 2D TMDs, where monolayer-rich dispersions are often required to exploit their interesting semiconducting properties. ${ }^{6}$ 
One solution to the above-stated problem may be to synthesise nanosheets using controlled 'bottom-up' rather than topdown approaches. One commonly used approach is chemical vapour deposition (CVD), which can produce highly crystalline, defect-free, large lateral size monolayers of TMDs and many other layered materials. ${ }^{12-14}$ The drawback of this method is that it is very expensive, and shows low potential for commercial scalability. Thus, new methods are required for the synthesis of TMDs, which could combine both the scalability and cost-effectiveness of LPE and the product quality of CVD. Solution-based "bottom-up" approaches may have the potential to synthesise TMDs in this way.

The main goal of this review is to analyse and discuss various methodologies for the synthesis of TMDs using solution-based "bottom-up" approaches. The properties, such as phase, morphology, and electrical properties, of the products resulting from each synthetic regime will be analysed, and the applications arising from these properties will be discussed. As much of the research on TMDs is dedicated to Group VI TMDs such as molybdenum and tungsten disulfides and diselenides, the main focus of this review will be on these materials. Finally, we will look at some challenges facing the field of bottom-up synthesis of TMDs, and finish by discussing some future prospects of TMD nanostructures synthesised via various "bottom-up" approaches.

\section{TMD polymorphs}

TMD monolayers consist of an atomic layer of transition metal atoms, M (such as Mo, W, etc.), sandwiched between 2 atomic
Trigonal prismatic

a)

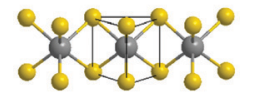

\section{Octahedral}

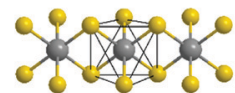

1T

b)

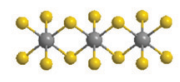

$2 \mathbf{H}$

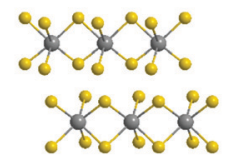

$3 \mathbf{R}$

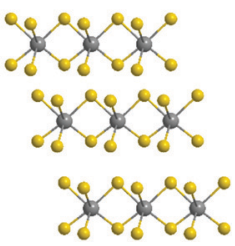

Fig. 1 (a) Trigonal prismatic and octahedral transition metal coordination in TMDs. (b) The layer stacking in the most common polytypes of TMDs - 1T, $2 \mathrm{H}$ and $3 \mathrm{R}$. The grey and yellow spheres represent transition metal and chalcogen atoms, respectively.

layers of chalcogen atoms, $\mathrm{X}$, (such as S, Se, Te, etc.). van der Waals interactions between these monolayers cause them to stack, forming bulk TMDs. Both the coordination of the transition metal atom and the stacking sequence of the monolayers can change, leading to the formation of different phases or polymorphs (Fig. 1), all of which can have different properties. The combination of the d-electron configuration and coordination of the transition metal are the predominant factors in determining its electrical and conductive properties.

There are 3 main phases of TMDs: the 1T, $2 \mathrm{H}$ and $3 \mathrm{R}$ phases. The distorted $1 \mathrm{~T}^{\prime}$ phase is also one of significant research

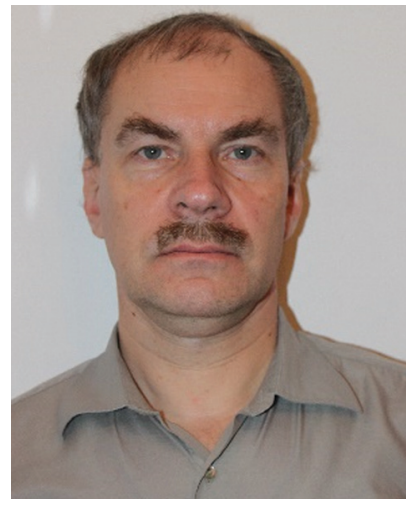

Yurii K. Gun'ko

Yurii Gun'ko received his MSc and $\mathrm{PhD}$ degrees in Chemistry from Moscow State University (Russia) in 1987 and 1990 respectively. Then he worked as a lecturer in Chemistry in Belarus Institute of Technology and as a postdoctoral researcher in the University of Sussex (UK). In 1995 he was awarded the Alexander von Humboldt fellowship of the University of Gottingen and Magdeburg (Germany). After that he returned back to the University of Sussex. In 1999 he was appointed as a Lecturer in Inorganic Chemistry in Trinity College Dublin, was promoted to Senior Lecturer and elected to Fellowship in 2005, became an Associate Professor in 2007 and was appointed to the 1922 Chair in 2014. He has a very wide experience in Inorganic Chemistry and has made a particular use of this knowledge to pioneer innovative routes to new materials, with potential applications in areas such as solar energy conversion, optical sensing, catalysis, magnetic materials (including those for MRI) and adhesive technology. His main research interest and activities are in the areas of quantum dot based materials, nanophotonics, photovoltaic cells, and plasmonic and magnetic nanostructures. 
interest. The phases or polymorphs of TMDs are named using numbers, which denote the number of repeating $\mathrm{X}-\mathrm{M}-\mathrm{X}$ layers in the unit cell, and letters, which describe their crystal system.

\section{$1 T$ phase}

This TMD polymorph has one $\mathrm{X}-\mathrm{M}-\mathrm{X}$ layer in its unit cell, which has trigonal symmetry with the atoms being arranged in an AbA AbA pattern. Capital letters and lower-case letters represent chalcogen and transition metal atoms respectively, and the letter itself represents a position in space. In the 1T TMD polymorph, the transition metal atom exists in an octahedral coordination environment. Group VI TMDs of this polytype, e.g. $1 \mathrm{~T}-\mathrm{MoS}_{2}$ and $\mathrm{WS}_{2}$, are metallic, as a result of partial filling of the $\mathrm{d}_{x y}, \mathrm{~d}_{x z}$ and $\mathrm{d}_{y z}$ set of degenerate orbitals that arise due to octahedral coordination (Fig. 2(a)).

\section{H phase}

The $2 \mathrm{H}$ polymorph belongs to the hexagonal crystal group. It involves the transition metal atom coordinated in a trigonal prismatic fashion. There are two repeating $\mathrm{X}-\mathrm{M}-\mathrm{X}$ units in its unit cell, with the atoms following an $\mathrm{AbA} \mathrm{BaB}$ AbA pattern. $2 \mathrm{H}$ TMDs from Group VI of the periodic table are semiconducting, due to complete filling of the lowest energy $d_{z^{2}}$ orbital that arises from trigonal prismatic coordination (Fig. 2(b)).

\section{R phase}

The $3 \mathrm{R}$ polytype is similar to the $2 \mathrm{H}$, in that it involves trigonal prismatic coordination of the transition metal. However, it differs in its stacking pattern. The unit cell involves 3 monolayers, stacked in an AbA BcB CaC AbA fashion. This polymorph belongs to the rhombohedral crystal group, and like the $2 \mathrm{H}$ phase, leads to semiconducting Group VI TMDs due to the trigonal prismatic coordination of the transition metal (Fig. 2(b)). ${ }^{15}$
It should be noted that, in the case of monolayer TMDs, there are only two phases - 1T and $1 \mathrm{H}$ - as the stacking sequence is no longer a factor.

\section{Bottom-up synthetic routes}

In recent years, much progress has been made in the synthesis of TMDs through solution-phase bottom-up processes. The properties of the as-prepared TMDs vary widely with the use of different synthetic regimes, precursors, and stabilising ligands, giving rise to TMDs with a wide range of morphologies, phases and applications. In this section, the various established synthetic routes will be discussed, as well as the properties of the resulting TMD materials.

\subsection{Hydrothermal and solvothermal methods}

Hydro/solvothermal methods refer to the synthesis of materials in closed vessels at high temperatures and pressures, carried out in water (hydrothermal) or other non-aqueous solvents (solvothermal). ${ }^{16,17}$ Hydro/solvothermal methods are commonly used in the synthesis of a variety of nanomaterials. They are also widely reported methods in the wet-chemical, bottomup synthesis of TMD-based nanomaterials.

The solvothermal and hydrothermal approaches to the synthesis of nanoscale TMDs date back to the years 1998 and 2000, respectively. Zhan et al. developed a solvothermal method for the production of nanocrystalline $\mathrm{MoS}_{2}$ using pyridine as the solvent, and $\mathrm{MoO}_{3}$ and elemental sulfur as the precursors. ${ }^{18}$ The use of this method resulted in the formation of thin platelets of semiconducting $2 \mathrm{H} \mathrm{MoS}$, with average diameters of $100 \mathrm{~nm}$. This was followed closely by Fan et al., who successfully synthesised nanocrystallites of $\mathrm{MoS}_{2}$ and $\mathrm{MoSe}_{2}$ for the first time via a hydrothermal method. ${ }^{19}$ Using $\mathrm{Na}_{2} \mathrm{MoO}_{4}$ as the Mo source and $\mathrm{Na}_{2} \mathrm{~S}_{2} \mathrm{O}_{3} / \mathrm{Na}_{2} \mathrm{SeSO}_{3}$ as the sulfur/selenium sources, amorphous $\mathrm{MoS}_{2}$ and $\mathrm{MoSe}_{2}$ were formed, of average crystallite sizes 4 and $7 \mathrm{~nm}$, respectively, which when annealed

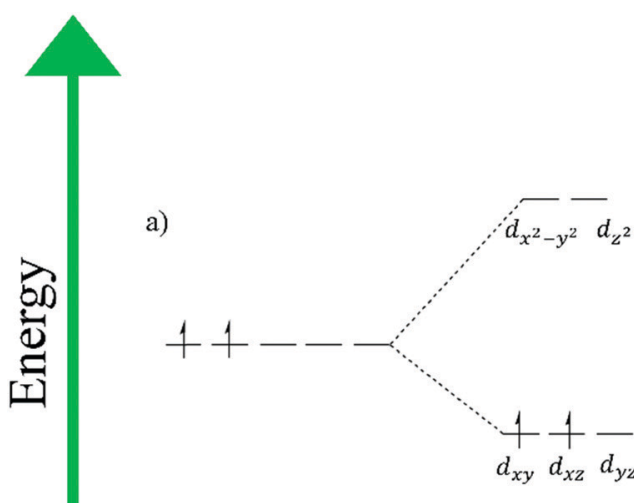

Octahedral splitting (eg: $1 \mathrm{~T} \mathrm{MoS}_{2}$ ) b)

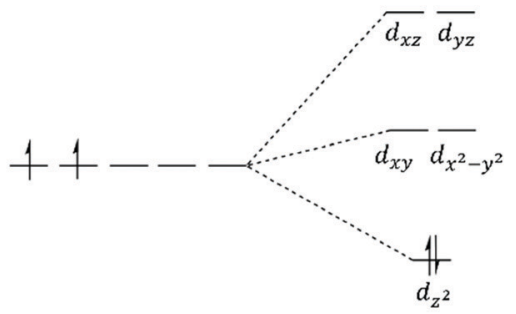

Trigonal prismatic splitting (eg: $2 \mathrm{H}, 3 \mathrm{R} \mathrm{MoS}_{2}$ )

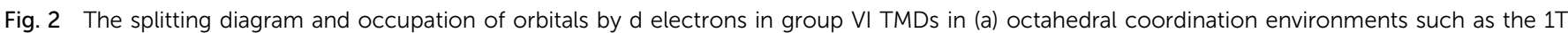
phase and (b) trigonal prismatic coordination environments such as the $2 \mathrm{H}$ and $3 \mathrm{R}$ phases. 


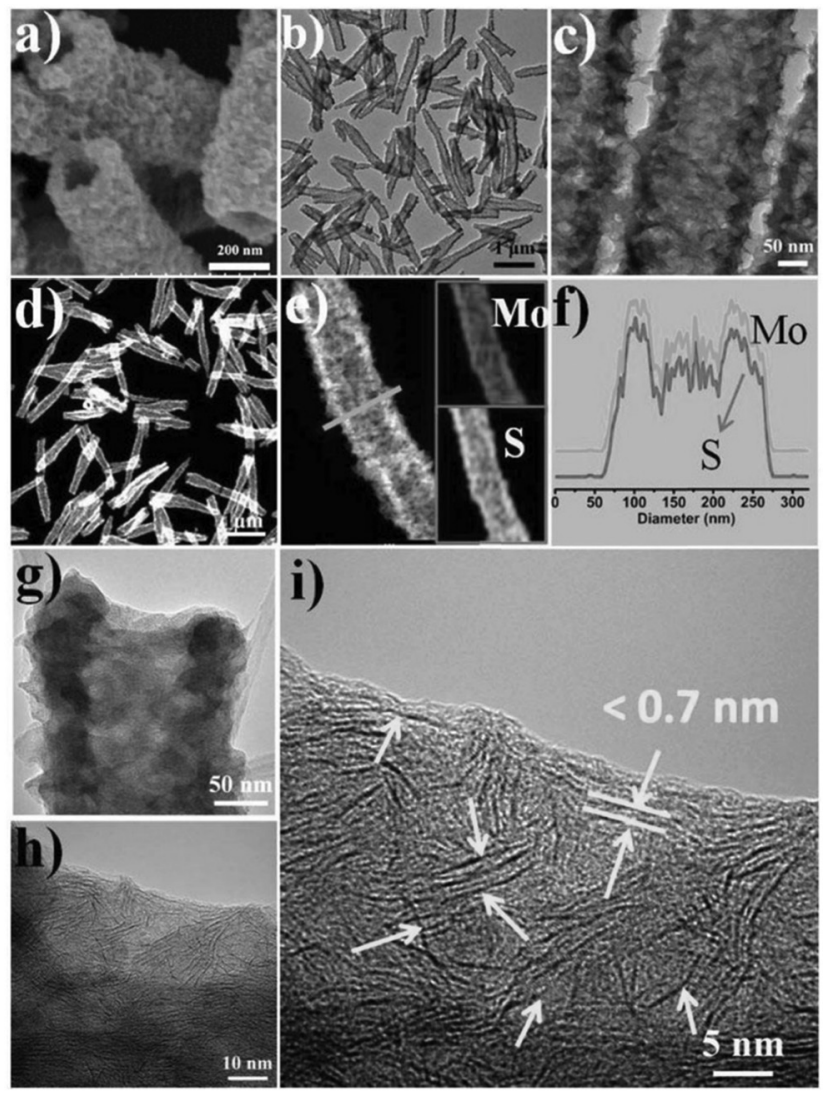

Fig. 3 (a) SEM, (b and c) TEM, and ( $d$ and e) STEM images of the 3D architectures assembled from single-layered $\mathrm{MoS}_{2}$. The insets in (e) are EDX elemental maps, which clearly indicate that molybdenum and sulphur are uniformly distributed in the assembled architectures. (f) Corresponding EDS line scan profiles for Mo and S along the line in image (e). (g) TEM image of a single tube. (h) HRTEM image of the rim of the tube in (g). (i) Enlarged image in $(\mathrm{g})$. The arrows indicate the separated layers with thickness below $0.7 \mathrm{~nm}$. Reproduced from ref. 23 .

at $350{ }^{\circ} \mathrm{C}$ for $9 \mathrm{~h}$ resulted in the formation of the semiconducting $2 \mathrm{H}$ phase, in the case of both $\mathrm{MoS}_{2}$ and $\mathrm{MoSe}_{2}$. The synthesis of both involved the use of hydrazine as a reducing agent for the reduction of $\mathrm{Mo}(\mathrm{vI})$ to $\mathrm{Mo}(\mathrm{Iv})$. These articles paved the way for the use of hydro/solvothermal methods in the bottom-up synthesis of ultrathin nanoscale TMDs.

Hydro/solvothermal synthesis of TMDs became very popular in the years that followed, producing nanostructures of various morphologies including nanosheets, nanotubes, nanorods, nanowires and nanoflowers. ${ }^{20-22}$

Wang et al. investigated the self-assembly behaviour of monolayer $\mathrm{MoS}_{2}$ synthesised via a simple solvothermal approach, using octylamine as the solvent and ethanol as an additive. ${ }^{23}$ In the absence of ethanol, $\mathrm{MoS}_{2}$ nanosheets were synthesised. However, in the presence of ethanol, hierarchical 3D tubular structures consisting of 2D nanosheets were formed (Fig. 3). The authors concluded that the formation of the tubular structure consisting of individual nanosheets, rather than a nanotube of restacked sheets, occurred due to a combination of factors. Firstly, ethanol in amine is a poor solvent, disrupting the interaction between nanosheets. Secondly, layered nanomaterials have a trend to form nanotubes under certain conditions. The octylamine capping ligands also prevent restacking of the sheets. The combination of these factors thus resulted in the formation of tubular arrangements of nanosheets, rather than single crystalline $\mathrm{MoS}_{2}$ nanotubes.

The authors also demonstrated that the diameter and length of the tubular structures could be tuned by changing the nature of alcohol present, using butanol as an example. It is also important to note that this synthesis is highly scalable, with $2 \mathrm{~g}$ of $\mathrm{MoS}_{2}$ hierarchical tubes being synthesised with ease, which is advantageous for potential commercial use.

\subsection{Hot-injection methods}

In the 1950s, LaMer and Dinegar proposed a set of conditions which are necessary to synthesise monodisperse colloidal solutions of nanoparticles, which involve a rapid nucleation step, followed by a slower step of controlled growth of nuclei to form larger particles. ${ }^{24}$ This motivated some groups of researchers to develop a new method of synthesising colloidal QDs, namely cadmium sulfide, selenide and telluride, which involved the rapid injection of organometallic precursors into vessels at high temperatures containing coordinating ligands and solvents. ${ }^{25}$ This method, termed 'hot-injection', has become commonplace in the synthesis of QDs, being used in the production of a wide variety of QDs, beyond just cadmiumbased QDs. $^{26,27}$

In recent years, significant efforts have been made to apply the method of hot-injection to the synthesis of monodisperse TMDs. Small variations in the method, such as stabilising ligands, precursors, reaction time and temperature, are shown to produce TMDs of various phases, sizes and morphologies.

3.2.1. TMD QDs. A natural evolution of the field of hot injection was to apply it to the synthesis of TMD QDs. Typically, TMD QDs are produced via LPE and post-processing, sizeselective centrifugation steps. ${ }^{28-30}$ However, these QDs often suffer from low quantum yield due to the presence of defects, and poor monodispersity, due to the lack of control of particle sizes associated with the LPE technique. ${ }^{31}$ However, Yin et al. produced blue-emitting $\mathrm{WS}_{2}$ QDs through a hot-injection approach. $^{32}$ The as-synthesised QDs exhibited an exceptionally high quantum yield - higher than previously reported in the literature. The synthesis involved the injection of thioacetamide into a vessel containing the metal precursor tungsten hexachloride $\left(\mathrm{WCl}_{6}\right)$, and coordinating ligands oleylamine (OAm) and oleic acid (OA) at various temperatures, 255, 275, and $295{ }^{\circ} \mathrm{C}$, to investigate the effects on the product.

The as-produced QDs were ultra-small lateral size, few-layer nanosheets (Fig. 4). The authors found that an increase in the reaction temperature corresponded to an increase in the diameter of the $\mathrm{WS}_{2}$ QDs, due to the increase in the reaction rate.

3.2.2. TMD nanoflowers. In 2019, Sokolikova et al. reported a hot-injection method for the synthesis of $\mathrm{WSe}_{2}$ nanoflowers exhibiting a less common, metastable phase - the $1 \mathrm{~T}^{\prime}$ phase. $^{33}$ The $1 \mathrm{~T}^{\prime}$ phase of TMDs arises from the distortion of the regular octahedral $1 \mathrm{~T}$ phase, and, in the case of $\mathrm{WSe}_{2}$, also results in metallic behaviour (Fig. 5). It should be noted that this phase is 

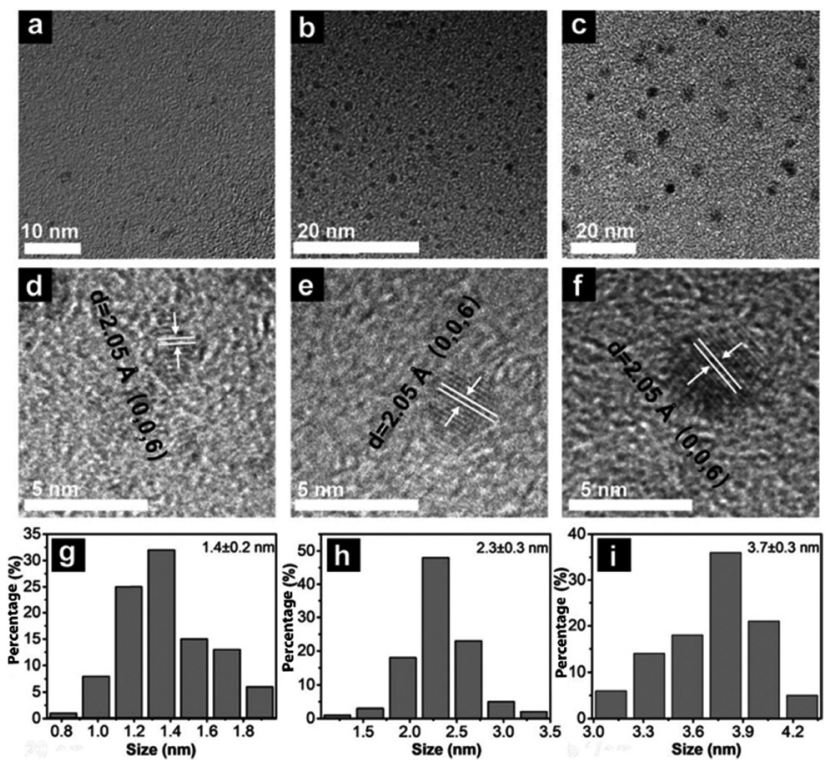

Fig. 4 TEM images of WS 2 QDs prepared at different reaction temperatures of (a) 255 , (b) 275 , and (c) $295^{\circ} \mathrm{C}$; (d-f) corresponding HRTEM images and $(\mathrm{g}-\mathrm{i})$ size distributions. Reproduced from ref. 32.

very difficult to consistently achieve through traditional methods of obtaining few layer TMDs, such as LPE. The authors achieved this with a hot-injection approach, involving the injection of a trioctylphosphine-selenium (TOP:Se) complex into a vessel containing $\mathrm{OA}$ and tungsten hexacarbonyl $\left(\mathrm{W}(\mathrm{CO})_{6}\right)$ as the metal precursor at $300{ }^{\circ} \mathrm{C}$ and for $3 \mathrm{~h}$.

The resulting nanostructures exhibited a flower-like morphology, of average diameter $200 \mathrm{~nm}$. The colloidal suspension of $\mathrm{WSe}_{2}$ nanoflowers in ethanol was observed to be stable under ambient conditions. The $1 \mathrm{~T}^{\prime}$ nanoflowers were also fully convertible to the semiconducting $2 \mathrm{H}$ phase via annealing at $400{ }^{\circ} \mathrm{C}$ for $2 \mathrm{~h}$, with no obvious changes in their size or morphology.

3.2.3. TMD nanosheets. Savjani et al. reported the synthesis of oleylamine (OAm)-stabilised semiconducting $1 \mathrm{H} \mathrm{MoS}$ by hot-injection using a single-source precursor (SSP), $\left[\mathrm{Mo}_{2} \mathrm{O}_{2} \mathrm{~S}_{2}\left(\mathrm{~S}_{2} \mathrm{COEt}\right)\right]{ }^{34} \mathrm{~A}$ solution of the SSP in OAm was injected into a vessel containing hot OAm. This was done at various temperatures and aliquots were taken from the vessel at various time-points to investigate the effect of reaction time and temperature on the products. In all cases, monolayers of oleylamine-stabilised $\mathrm{MoS}_{2}$ were synthesised, which assembled into randomly oriented nanosheets encapsulated in an oleylamine matrix, with lateral sizes ranging from hundreds to thousands of nanometres (Fig. 6). The effect of reaction time on the nanosheets was found to be minimal, with the majority of nanosheet growth reaching completion after $3 \mathrm{~min}$. The reaction temperature, however, was shown to have an effect, with the products obtained at lower temperatures $\left(200{ }^{\circ} \mathrm{C}\right)$ having smaller average lateral dimensions $(4.78 \mathrm{~nm})$ compared with those obtained at higher temperatures $\left(325^{\circ} \mathrm{C}, 11.29 \mathrm{~nm}\right)$.

Jung et al. were able to produce size-controlled $\mathrm{MoSe}_{2}$ and $\mathrm{WSe}_{2}$ nanosheets using capping ligands with various binding energies to promote lateral growth of the nanosheets. ${ }^{35}$ Using $\mathrm{Mo}(\mathrm{CO})_{6} / \mathrm{W}(\mathrm{CO})_{6}$ and diphenyl diselenide $\left(\mathrm{Ph}_{2} \mathrm{Se}_{2}\right)$ as precursors, they varied the capping ligand used in the synthesis from oleylamine to oleyl alcohol to oleic acid. It was found that the use of the amine resulted in small-lateral size, multi-layered sheets, the alcohol resulted in fewer layers and a larger lateral size, and the carboxylic acid resulted in the production of large lateral size (200-400 nm) monolayers (Fig. 7).

Using DFT calculations, the researchers were able to show the significance of the binding energy of the capping ligand to the edge sites of $\mathrm{MoSe}_{2}$ and $\mathrm{WSe}_{2}$, and the effect this had on lateral size and thickness. It was demonstrated that the binding energy decreases on moving from the amine, to the alcohol, to the carboxylic acid. The strongly bound oleylamine stabilises the edge sites, favouring the formation of more layers and inhibiting lateral growth, whereas the weakly bound oleic acid does not offer much stabilisation to the edge sites of $\mathrm{MoSe}_{2}$ and $\mathrm{WSe}_{2}$, so lateral growth is favoured in this case. This insight into the factors controlling anisotropic growth in 2D materials may be a huge step forward for the solution-based bottom-up synthesis of these materials.

More recently, Zhou et al. successfully produced colloidal solutions of few-layer semiconducting $2 \mathrm{H} \mathrm{WS}_{2}$, using an array of capping ligands - oleylamine (OAm), oleic acid (OA), trioctylphosphine oxide (TOPO), and squalane. ${ }^{36}$ Raman spectroscopy is commonly used for characterisation of Group VI TMDs such as $\mathrm{MoS}_{2}$ and $\mathrm{WS}_{2}$ to determine the number of monolayers present in the sample. The frequencies of the $\mathrm{A}_{1 \mathrm{~g}}$ out-of-plane chalcogen vibrational mode, and the $\mathrm{E}_{2 \mathrm{~g}}^{1}$ vibrational mode, a
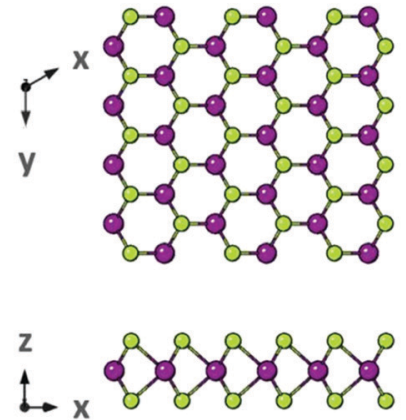

b

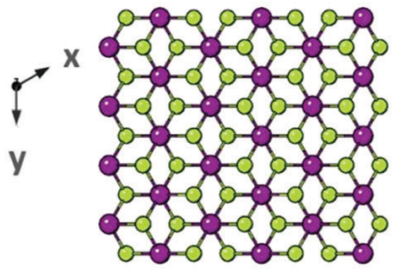

Z

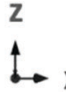

C
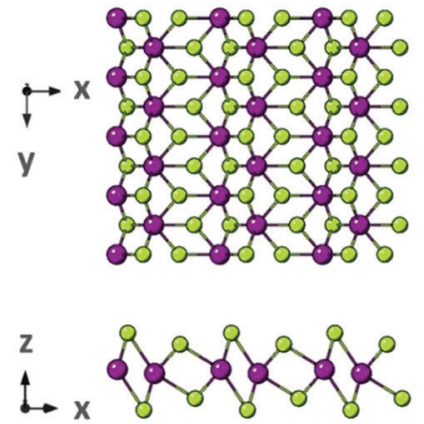

Fig. 5 Structural polymorphs of WSe $e_{2}$. (a) $2 \mathrm{H}$ WSe $e_{2}$ (space group $P 6_{3} / m m c$ ). (b) $1 T$ WSe $e_{2}$ (space group $P \overline{3} m 1$ ). (c) $1 T^{\prime} W S e_{2}$ (space group $P 2_{1} / m$ ). Reproduced from ref. 33. 

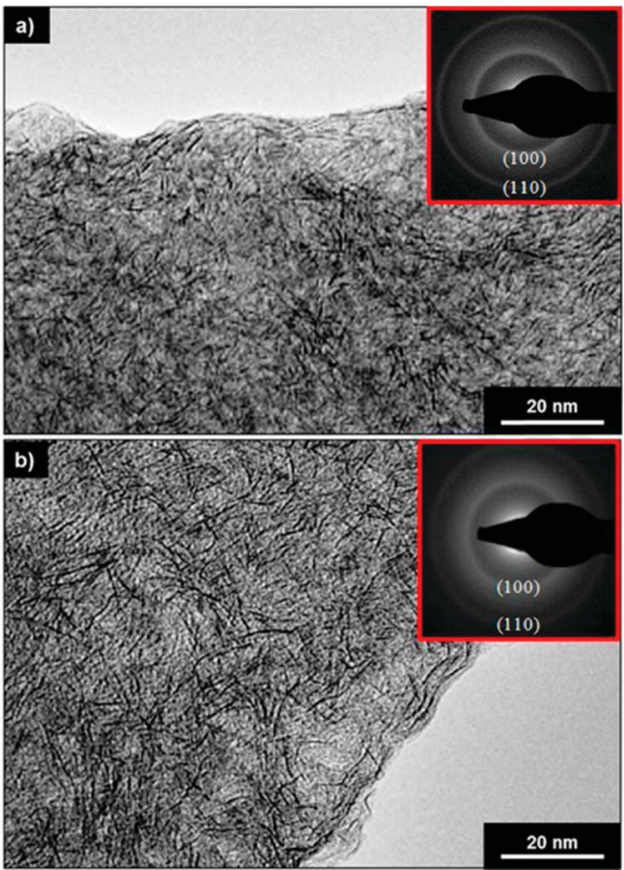

Fig. 6 TEM images of the $1 \mathrm{H}-\mathrm{MoS}_{2}$ @oleylamine flocculates give evidence for the presence of monolayer $\mathrm{MoS}_{2}$ nanosheets. The variation of the average nanosheet dimension from the reactions carried out at (a) $200^{\circ} \mathrm{C}$ (average size of $4.78 \pm 0.78 \mathrm{~nm}$ ) and (b) $325^{\circ} \mathrm{C}$ (average size of $11.29 \pm$ $1.26 \mathrm{~nm}$ ). The inserted images represent the SAED patterns, supporting the identification of the $1 \mathrm{H}$ crystallites. Reproduced from ref. 34 .

which involves the in-plane displacement of transition metal and chalcogen atoms, are monitored. The Raman shift between these two phonon modes has a thickness dependence - it decreases with decreasing number of monolayers - allowing the thickness of a sample to be determined..$^{37,38}$ The asproduced nanosheets were confirmed to have a thickness of 2 to 3 layers by AFM and Raman spectroscopy, and were reported to be of higher aspect ratio than those obtained by LPE combined with size-selective, post-processing steps.

It is clear that modification of the reaction conditions in the hot-injection approach - precursors, reaction temperatures and times, coordinating ligands, etc. - can result in the production of TMDs of a wide range of morphologies. The effects of changing these conditions need to be further understood in order for the hot-injection approach to be used widely in the synthesis of TMDs. In 2019, Geisenhoff $e t$ al. carried out a study on how ligands affect different properties of $\mathrm{WSe}_{2}$ nanocrystals, synthesised via hot-injection. ${ }^{39}$ The study involved the injection of a selenium precursor, $\mathrm{Ph}_{2} \mathrm{Se}_{2}$, into a mixture of tungsten hexacarbonyl $\left[\mathrm{W}(\mathrm{CO})_{6}\right]$ and two ligands - OA and TOPO. It was found that, by altering the ratio of $\mathrm{OA}$ and the tungsten precursor, the reactivity of the reagents changed drastically resulting in huge differences in the phases and morphologies of $\mathrm{WSe}_{2}$ products prepared under different conditions (Fig. 8).

\subsection{Other miscellaneous synthetic methods}

Although the hydrothermal and hot-injection methods are the most widely reported in the solution-based bottom-up synthesis of TMDs, there are some other interesting colloidal synthetic methods available in the literature.

3.3.1. Shape-transformation. For example, both Seo et al. and later Mastria et al. used a 'shape-transformation' procedure for the production of colloidal solutions of ultrathin sheets of $\mathrm{WS}_{2}$ via the sulfidation of tungsten oxide nanorods. ${ }^{40,41}$ Using a hot injection approach, with tungsten oxide $\left(\mathrm{W}_{18} \mathrm{O}_{49}\right)$ nanorods as the metal precursor, Seo et al. injected carbon disulfide into a solution of hexadecylamine and $\mathrm{W}_{18} \mathrm{O}_{49}$ nanorods at $250{ }^{\circ} \mathrm{C} .{ }^{40}$ The proposed reaction mechanism is through a 'rolling-out' process, by which $\mathrm{W}_{18} \mathrm{O}_{49}$ is gradually converted to $\mathrm{WS}_{2}$ via sulfidation, and the strain due to the heterogeneous phases results in the nanorod unravelling to form nanosheets (Fig. 9). The hexadecylamine acts as both a high-boiling point solvent and a stabilising ligand. This resulted in bundles of lateral-size controlled 2H-phase $\mathrm{WS}_{2}$ nanosheets.

Mastria et al. followed a very similar approach in the production of $\mathrm{WS}_{2}$ thin films via hot-injection, followed by spin-coating, with some slight modifications. ${ }^{41}$ Oleylamine was used in place of hexadecylamine as the coordinating solvent, and elemental sulfur was used in place of $\mathrm{CS}_{2}$ for the sulfidation step. The oleylamine seemed to have provided superior colloidal stability compared to hexadecylamine as used by Seo et al., evidenced by the fact that no major restacking of the sheets was observed in the TEM images. The ultrathin nature of these oleylamine-stabilised $\mathrm{WS}_{2}$ nanosheets was shown by the low electron diffraction contrast under low magnification TEM imaging.

3.3.2. One-pot method. Ding et al. synthesised a range of TMD QDs - MoS $, \mathrm{WS}_{2}, \mathrm{RuS}_{2}, \mathrm{MoTe}_{2}, \mathrm{MoSe}_{2}, \mathrm{WSe}_{2}$ and $\mathrm{RuSe}_{2}-$ via a simple, scalable, room-temperature, biomineralizationassisted "one-pot" approach, using deuterated water as the reaction medium. ${ }^{42}$ Using bovine serum albumin (BSA) as a biocompatible surfactant, the resultant $3.9 \mathrm{~nm}$ diameter $2 \mathrm{H}-\mathrm{MoS}_{2}$ QDs were shown to be stable in aqueous suspension after 3 months, which is vital to be suitable in biomedical applications. The authors also produced a variety of other biomolecule-stabilised $\mathrm{MoS}_{2}$ QDs, using gluconate, polyarginine and cysteine. However, the BSA-stabilised $\mathrm{MoS}_{2}$ resulted in the synthesis of smaller, more monodisperse QDs. This was shown to be due to the presence of disulfide bonds in BSA, which have a much higher binding energy to $\mathrm{MoS}_{2}$ QDs compared to other functional groups such as thiol, carboxylic acid and hydroxy groups. It is worth noting that this method is very time efficient, with the synthesis taking only 10-20 s, making it a much more attractive method for the synthesis of TMD QDs compared to traditional, LPE-based methods, which can typically take on the order of $24 \mathrm{~h}^{43}$

3.3.3. Calcination. One of the lesser explored routes for the production of TMDs is calcination. Sharma et al. developed a multi-step bottom-up process for the production of colloidal $\mathrm{WS}_{3}$, which when subjected to calcination at $850{ }^{\circ} \mathrm{C}$, transforms into $\mathrm{WS}_{2}$ nanosheets. ${ }^{44}$ Although not as simple as hydrothermal or hot-injection processes which often only involve one or two steps, this bottom-up synthetic process is advantageous for multiple reasons. Firstly, it does not involve the introduction of 
a

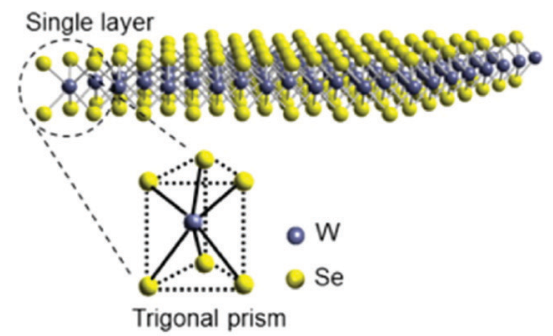

b

$\mathrm{W}(\mathrm{CO})_{6}+2 \mathrm{Ph}_{2} \mathrm{Se}_{2} \underset{\substack{\text { Capping } \\ \text { ligand }}}{\longrightarrow} \mathrm{WSe}_{2}+2 \mathrm{Ph}_{2} \mathrm{Se}+6 \mathrm{CO}$

e

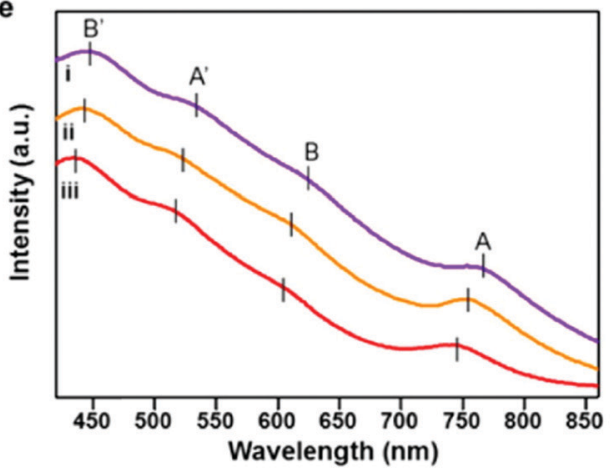

c
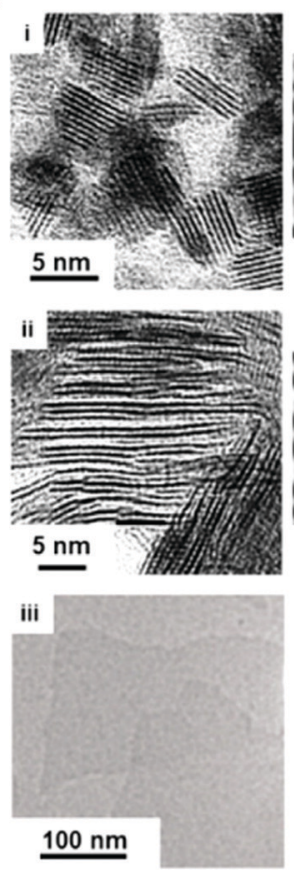

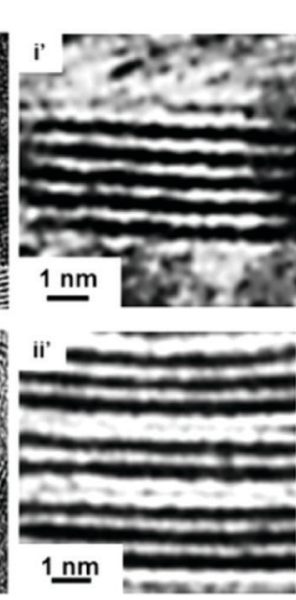

iii'

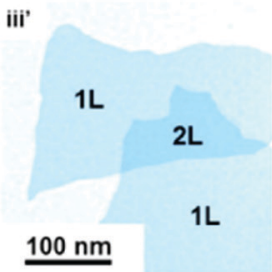

d

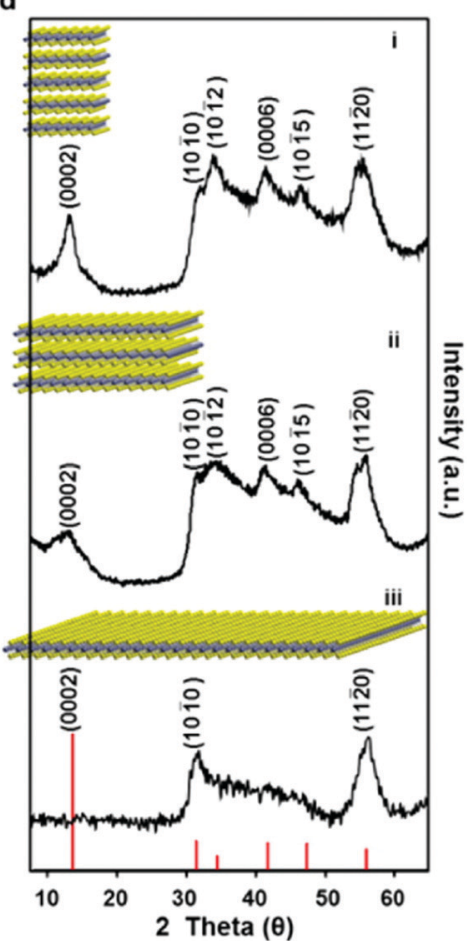

Fig. 7 Lateral size- and thickness-controlled synthesis of WSe $\mathrm{S}_{2}$ nanosheets with the use of different capping ligands. (a) Visualization of single-layer $W \mathrm{Se}_{2}$ and its trigonal prism geometry with the ball-and-stick model. (b) Chemical equation for WSe $\mathrm{W}_{2}$ nanosheets. (c) TEM images of WSe 2 nanosheets synthesized with (i) oleylamine, (ii) oleyl alcohol, and (iii) oleic acid. (i' and ii') Magnified TEM images of the nanosheets, and (iii') pseudocolor image of the single-layer nanosheets. (d) XRD patterns of $\mathrm{WSe}_{2}$ nanosheets synthesized with (i) oleylamine, (ii) oleyl alcohol, and (iii) oleic acid. (e) UV-vis absorption spectra of $\mathrm{WSe}_{2}$ nanosheets synthesized with (i) oleylamine (purple line), (ii) oleyl alcohol (orange line), and (iii) oleic acid (red line). Reproduced from ref. 35 .

surfactants to stabilise the nanosheets, which can affect some of the material properties, such as conductivity. Secondly, the product consists of monodisperse hexagonal sheets of pure semiconducting $\mathrm{WS}_{2}$, composed of both $2 \mathrm{H}$ and $3 \mathrm{R}$ phase, and contains no metallic $1 \mathrm{~T}$ or $1 \mathrm{~T}^{\prime}$ phase. Finally, and most importantly, the process is highly scalable. The crystallinity and purity of the sample should not vary with scale, allowing for this process to have potential industrial scale applications. The authors provided proof of this by scaling up their initial synthesis 16 -fold and producing $12 \mathrm{~g}$ of dry $\mathrm{WS}_{2}$ nanosheets.

It is clear that the bottom-up synthesis of $2 \mathrm{D}$ TMDs is an exciting new field. The various techniques employed to make these materials are very versatile, and result in a wide variety of high quality TMDs, with varying sizes, phases, morphologies and other properties, with relative ease. The various synthetic techniques are summarised in Table 1.

\section{Applications}

The benefit of the above-mentioned synthetic techniques is that they mainly give rise to phase-pure, monodisperse products, and that the phase, size and morphology can often be tuned by modification of the reaction conditions. TMDs have a wide variety of already-established applications, as well as potential applications, which are heavily dependent on their size, phase and morphology. In this section, some of the main applications of TMDs - biomedical, electronics and catalysis will be discussed, and the importance of 'bottom-up' synthetic routes for achieving these applications will be highlighted.

\subsection{Biomedical applications}

TMD nanostructures possess many unique and interesting properties which make them promising candidates for use in biomedical applications. Semiconducting 2D TMDs, such as $\mathrm{MoS}_{2}$ and $\mathrm{WS}_{2}$, have a direct bandgap, with monolayer $\mathrm{MoS}_{2}$ for example exhibiting strong photoluminescence at 627 and $677 \mathrm{~nm}$, making them exciting candidates for use in biosensing and bioimaging. ${ }^{6,59}$ The exceptionally high surface-to-volume ratio of TMD nanosheets is also advantageous, as this is a key factor in choosing suitable nanomaterials for drug delivery. ${ }^{60}$ However, one drawback is that they typically exhibit poor stability in aqueous media, which can limit their potential use in biomedical applications. ${ }^{61}$ Surface functionalisation with suitable surfactants or biomolecules can overcome this problem. The benefit of bottom-up synthetic methods such as hydro/solvothermal and hot injection syntheses is that they involve the use of suitable surfactants or ligands that coordinate to and stabilise the nanomaterial. Therefore, wet-chemical bottom-up approaches to the synthesis of TMDs can allow for the material to be synthesised and stabilised/functionalised in 


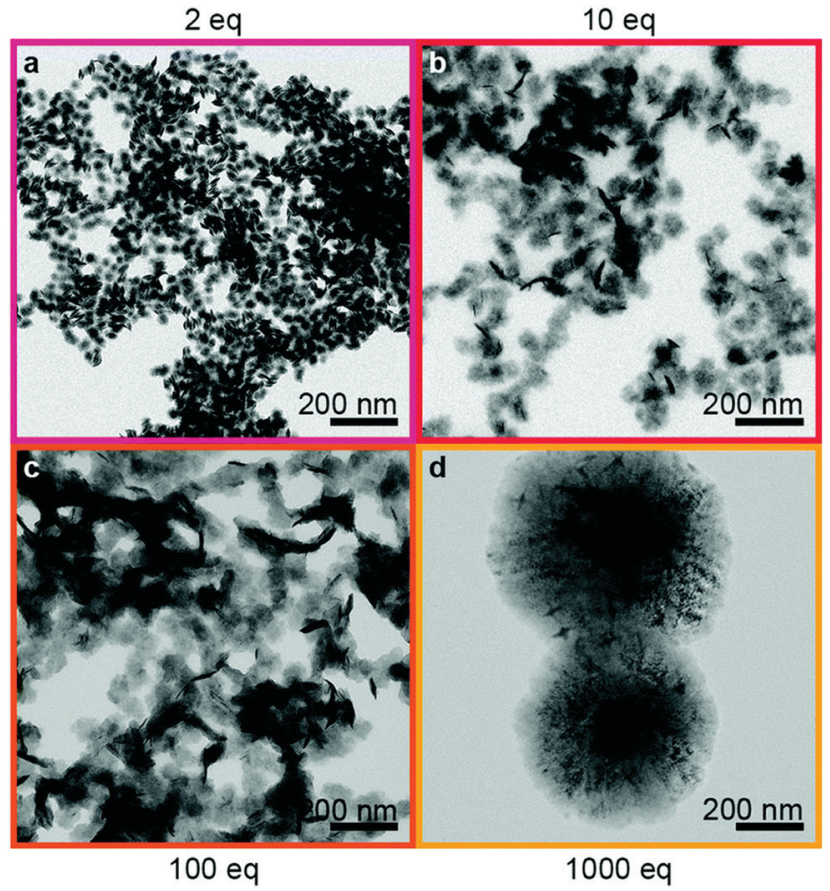

Fig. 8 TEM images of $\mathrm{WSe}_{2}$ nanocrystals synthesised via injection of $1 \mathrm{~mL}$ of $\mathrm{Ph}_{2} \mathrm{Se}_{2}\left(0.04 \mathrm{M}\right.$ in TOPO or OA) into a solution of $\mathrm{W}(\mathrm{CO})_{6}(0.02 \mathrm{mmol})$ in OA/TOPO. The final reaction mixtures contained (a) 2, (b) 10, (c) 100 or (d) 1000 eq. OA/W. Reproduced from ref. 39.

the same process. Furthermore, these wet-chemical synthetic approaches often result in the production of more monodisperse, well-controlled TMD nanostructures than those obtained via top-down approaches, which is vital in ensuring consistency for biomedical applications.

PEGylation is a functionalisation method commonly used to make nanomaterials more suitable for biomedical applications. It involves the attachment of polyethylene glycol (PEG) molecules to other entities. ${ }^{62}$ PEGylated nanomaterials have the advantage of better water solubility, reduced immunogenicity when introduced into the body, and prolonged blood circulation time. ${ }^{63}$

Kumar et al. developed PEGylated $\mathrm{MoS}_{2}$ microspheres via a hydrothermal method for use as potential anti-cancer agents. ${ }^{43}$ Using ammonium heptamolybdate tetrahydrate and thiourea as precursors, and the addition of PEG as a stabilising ligand, they successfully produced $2-4 \mu \mathrm{m}$ microspheres, consisting of bundles of ultrathin sheets of PEGylated $2 \mathrm{H} \mathrm{MoS}_{2}$. The authors found, through altering the concentration of PEG used in the reaction, that PEG played a vital role in the self-assembly of the PEGylated $\mathrm{MoS}_{2}$ to form the hierarchical micro-spherical structure. These PEGylated $\mathrm{MoS}_{2}$ microstructures were shown to have excellent cytotoxicity towards cells from the MCF-7 breast cancer cell line, due to PEGylation aiding in the cellular uptake of $\mathrm{MoS}_{2}$. It is worth nothing that $\mathrm{MoS}_{2}$ nanostructures have been previously shown to exhibit low toxicity towards normal cells. These 'bottom-up' synthesised $\mathrm{MoS}_{2}$ nanostructures, thus have the capability to act simultaneously as both imaging/ sensing and anti-cancer agents.

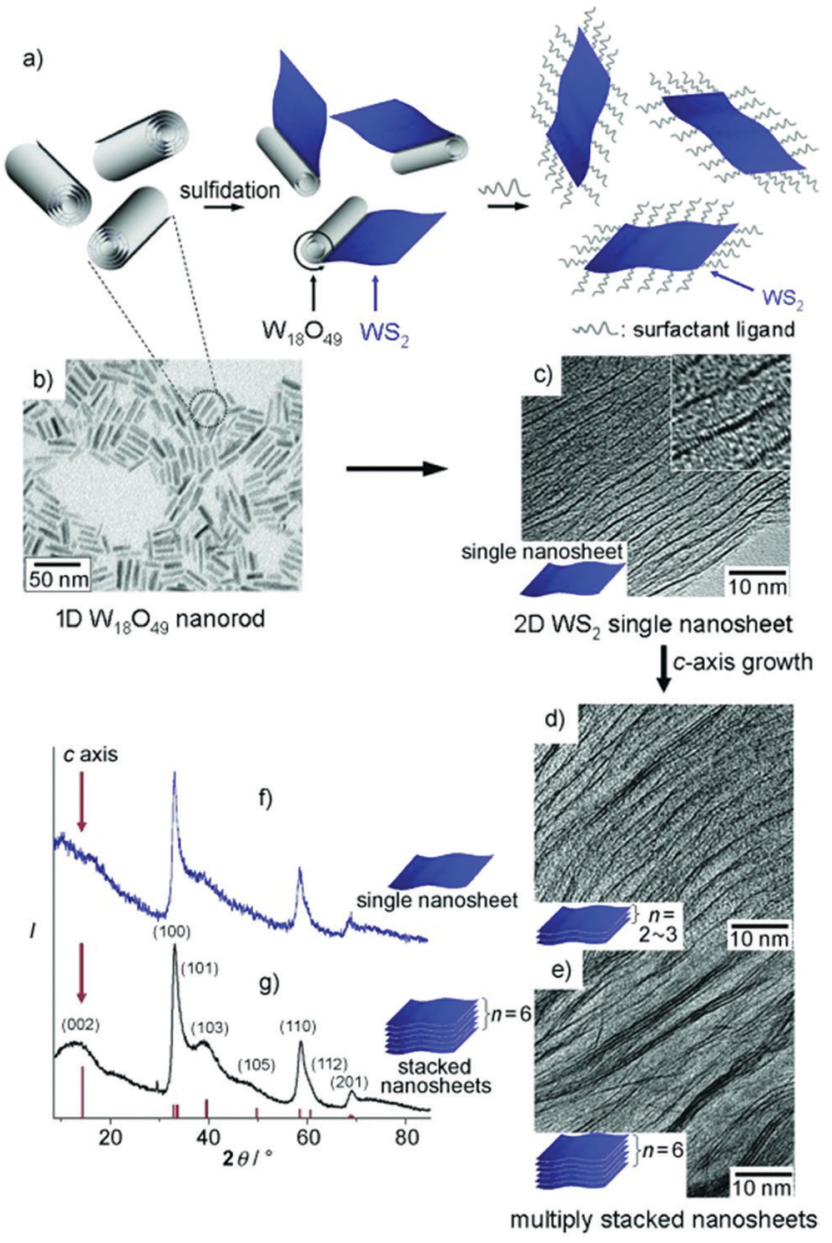

Fig. $92 \mathrm{D} \mathrm{WS}$ nanosheet crystal formation through rolling-out shapetransformation processes of $1 \mathrm{D} \mathrm{W} \mathrm{W}_{18} \mathrm{O}_{49}$ nanorod precursors. (a) Schematic diagram for the synthesis of 2D WS 2 nanosheet crystals. (b) TEM image of $\mathrm{W}_{18} \mathrm{O}_{49}$ nanorod precursors. (c-e) TEM images of 2D WS 2 obtained with the reaction times of $10 \mathrm{~min}$ (c), $30 \mathrm{~min}$ (d), and $1 \mathrm{~h} \mathrm{(e)} \mathrm{after} \mathrm{the} \mathrm{CS}_{2}$ injection. (f) XRD analyses of the $\mathrm{WS}_{2}$ single (top) and stacked (bottom) nanosheet crystals (red lines: JCPDS reference card no. 08-0237). Reproduced from ref. 40 .

One particularly exciting potential application of TMD nanostructures is as agents for photodynamic (PDT) and photothermal therapy (PTT). ${ }^{64,65}$ PDT is an anti-cancer treatment, and involves the use of light and photosensitive species. When the photosensitive compound is in the body, under conditions of no light irradiation, it is non-toxic. However, when the photosensitive compound is exposed to light of correct wavelength, it is activated, producing reactive oxygen species (ROS). ROS are toxic to cells, therefore inducing cell death in the targeted area. ${ }^{66}$ PTT is a type of PDT which does not require the generation of ROS. Instead, light irradiation (usually infrared) of the photosensitive species triggers the release of heat, killing the targeted cells.

Wang et al. produced $\mathrm{MoSe}_{2}$ nanoflowers via a hot injection approach for potential use as PTT/PDT agents, using sodium molybdate and selenium powder as transition metal and chalcogen sources, respectively. ${ }^{56}$ The resulting semiconducting $2 \mathrm{H}$ 
Table 1 Comparison of different synthetic techniques for the production of various TMD materials

\begin{tabular}{|c|c|c|c|c|c|c|}
\hline Method & TMD materials & Morphologies & Phases & Advantages & Disadvantages & Ref. \\
\hline Hydrothermal & $\begin{array}{l}\mathrm{MoS}_{2}, \mathrm{MoSe}_{2} \\
\mathrm{WS}_{2}, \mathrm{WSe}_{2}\end{array}$ & $\begin{array}{l}\text { Microrods, micro/nanoparticles, } \\
\text { microspheres, nanosheets, } \\
\text { nanoflowers, laterally confined } \\
\text { monolayers (QDs) }\end{array}$ & $\begin{array}{l}\text { Amorphous, } \\
2 \mathrm{H}, 1 \mathrm{~T} / 1 \mathrm{~T}^{\prime}\end{array}$ & $\begin{array}{l}\text { Simple, one-step process, } \\
\text { aqueous phase synthesis }\end{array}$ & $\begin{array}{l}\text { High temperatures } \\
\text { and pressures } \\
\text { required }\end{array}$ & $\begin{array}{l}19-21 \\
\text { and } \\
45-55\end{array}$ \\
\hline Solvothermal & $\mathrm{MoS}_{2}, \mathrm{WS}_{2}$ & $\begin{array}{l}\text { Platelets, nanosheets, 3D } \\
\text { self-assembled tubes }\end{array}$ & $2 \mathrm{H}$ & Simple, one-step process & $\begin{array}{l}\text { Requires solvents, } \\
\text { may be hard to } \\
\text { remove from the } \\
\text { final product }\end{array}$ & $\begin{array}{l}18,22 \\
\text { and } \\
23\end{array}$ \\
\hline Hot injection & $\begin{array}{l}\mathrm{MoS}_{2}, \mathrm{MoSe}_{2} \\
\mathrm{WS}_{2}, \mathrm{WSe}_{2}\end{array}$ & $\begin{array}{l}\text { Laterally confined monolayers } \\
\text { (QDs), nanoflowers, monolayer } \\
\text { and few-layer nanosheets }\end{array}$ & $1 \mathrm{H}, 2 \mathrm{H}, 1 \mathrm{~T}^{\prime}$ & $\begin{array}{l}\text { High level of controllability, } \\
\text { monodisperse products }\end{array}$ & $\begin{array}{l}\text { High temperatures, } \\
\text { solvents may be } \\
\text { hard to remove } \\
\text { from the final } \\
\text { product }\end{array}$ & $\begin{array}{l}32- \\
36,39 \\
\text { and } \\
56-58\end{array}$ \\
\hline $\begin{array}{l}\text { One-pot } \\
\text { method }\end{array}$ & $\begin{array}{l}\mathrm{MoS}_{2}, \mathrm{WS}_{2} \\
\mathrm{RuS}_{2} \mathrm{MoTe}_{2} \\
\mathrm{MoSe}_{2}, \mathrm{WSe}_{2} \\
\mathrm{RuSe}_{2} \text { QDs }\end{array}$ & $\begin{array}{l}\text { Laterally confined monolayers } \\
\text { (QDs) }\end{array}$ & $2 \mathrm{H}$ & $\begin{array}{l}\text { Incredibly fast reaction } \\
\text { time, uniform, monodisperse } \\
\text { product, a wide variety } \\
\text { of TMDs possible }\end{array}$ & - & 42 \\
\hline $\begin{array}{l}\text { Shape- } \\
\text { transformation }\end{array}$ & $\begin{array}{l}\mathrm{WS}_{2} \text { ultrathin } \\
\text { nanosheets }\end{array}$ & $\begin{array}{l}\text { Ultrathin nanosheets, } \\
\text { stacked nanosheets }\end{array}$ & $1 \mathrm{~T}^{\prime}, 2 \mathrm{H}$ & $\begin{array}{l}\text { Highly monodisperse } \\
\text { product }\end{array}$ & $\begin{array}{l}\text { Multi-step process, } \\
\text { requires high } \\
\text { quality } \mathrm{W}_{18} \mathrm{O}_{49} \\
\text { nanorods as } \\
\text { precursors }\end{array}$ & $\begin{array}{l}40 \\
\text { and } \\
41\end{array}$ \\
\hline Calcination & $\mathrm{WS}_{2}$ & Nanosheets & $2 \mathrm{H}, 3 \mathrm{R}$ & $\begin{array}{l}\text { Highly scalable, no use of } \\
\text { surfactants required, allows } \\
\text { for large quantities of } \\
\text { nanomaterials to be produced }\end{array}$ & $\begin{array}{l}\text { Multi-step } \\
\text { process, very } \\
\text { high temperatures } \\
\text { required }\end{array}$ & 44 \\
\hline
\end{tabular}

$\mathrm{MoSe}_{2}$ nanoflowers, which consisted of $150 \mathrm{~nm}$ diameter bundles of sheets, each 3-4 layers thick, displayed a strong absorbance in the NIR region, at $808 \mathrm{~nm}$. These $\mathrm{MoSe}_{2}$ nanoflowers showed high photothermal stability, and demonstrated superior photothermal conversion efficiency (61.8\%) compared to similar previously synthesised $\mathrm{MoSe}_{2}$-based PTT agents, such as PVP-coated $\mathrm{MoSe}_{2}$ nanosheets (57.9\%) and $\mathrm{MoSe}_{2} @ P D A$ nanocomposites (44.5\%). ${ }^{67,68}$ The high photothermal conversion efficiency of these $\mathrm{MoSe}_{2}$ nanoflowers can be attributed to their branched, nanoflower-like morphology, which is readily achievable only through solution-based bottom-up synthetic strategies such as hydro/solvothermal and hot-injection syntheses. Furthermore, the $\mathrm{MoSe}_{2}$ nanoflowers were found to be effective in producing reactive oxygen species, necessary for PDT. These nanoflowers were subsequently PEGylated for further experiments to increase biocompatibility, and these PEG-MoSe ${ }_{2}$ nanohybrids were loaded with an anticancer drug, Doxorubicin (Dox), to investigate their loading capacity and potential for controlled drug release (Fig. 10). The nanoflowers were found to have a high drug loading capacity of Dox $(12.6 \pm 2.5 \mathrm{wt} \%)$, with inhibited drug release at $\mathrm{pH}$ 7.4, and enhanced release of Dox under acidic conditions of $\mathrm{pH} 5$, which is significant as tumour sites are weakly acidic, displaying $\mathrm{pH}$ values of around 4-5. Therefore, the authors, through a simple bottom-up synthetic approach, produced $\mathrm{MoSe}_{2}$-based nanostructures with strong potential for use as PTT/PDT/chemotherapy agents.

In addition to potential anti-cancer applications, some recent studies have also focused on the potential of TMD nanomaterials as antibacterial agents. Masimukku et al. synthesised $<1 \mu \mathrm{m}$ wide, single-to-few layer $\mathrm{WS}_{2}$ nanoflowers via a hydrothermal method, using sodium tungsten dihydrate and thiocarbamide as tungsten and sulfur sources, respectively (Fig. 11). ${ }^{46}$
Through piezoresponse force microscopy (PFM), the resultant metallic $1 \mathrm{~T} \mathrm{WS}_{2}$ nanoflowers were shown to have a piezoelectric response. This piezoresponse was examined further to see if the $\mathrm{WS}_{2}$ nanoflowers exhibited piezo-degradation ability towards Rhodamine-B (Rh-B) dye. $\mathrm{WS}_{2}$ nanoflowers were dispersed in a solution of Rh-B and subjected to ultrasonication in the dark for $200 \mathrm{~s}$, and were found to exhibit 99\% degradation of Rh-B. As a control, $\mathrm{WS}_{2}$ nanoflowers were dispersed in Rh-B for $200 \mathrm{~s}$ in the dark and in the absence of ultrasonication, and

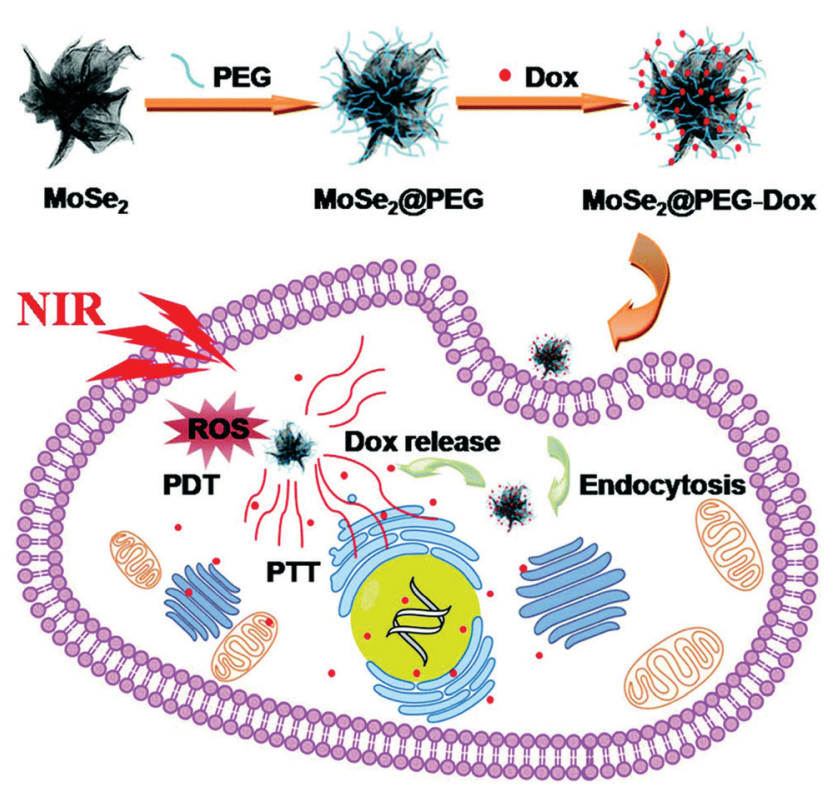

Fig. 10 Schematic representation of the preparation and therapeutic action of $\mathrm{MoSe}_{2}$ @PEG-Dox nanocomposites. Reproduced from ref. 56. 


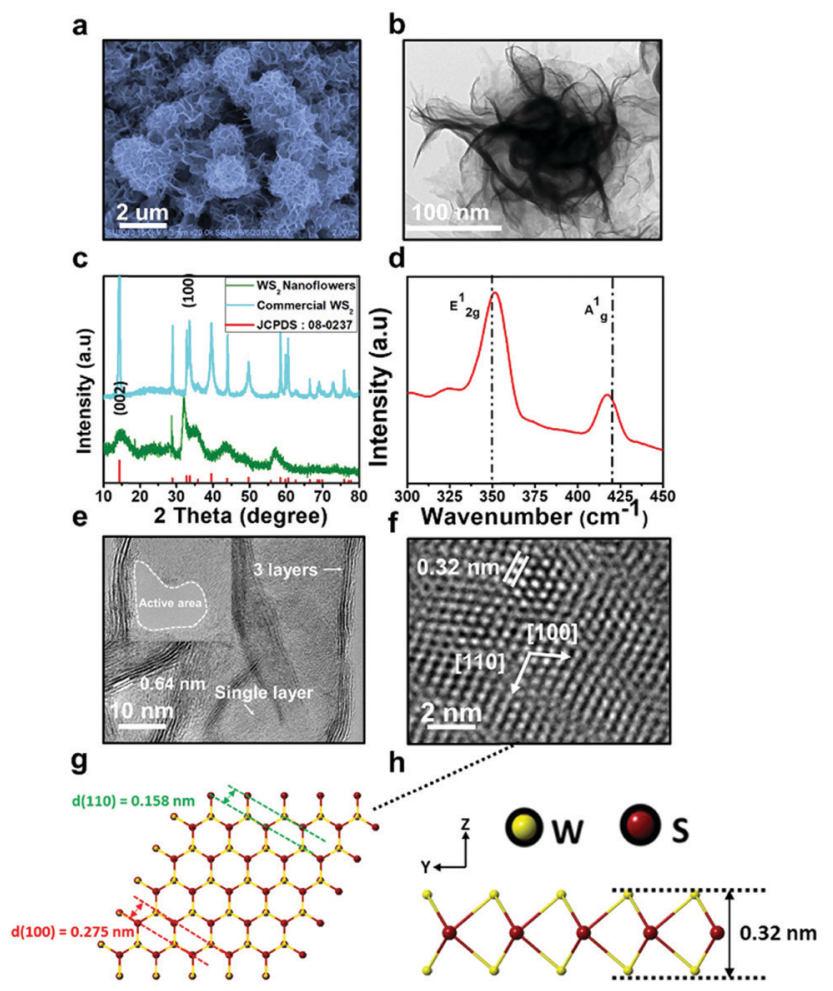

Fig. 11 (a) FESEM image of the WS 2 NFs showing the size in the range of $0.5 \mu \mathrm{m}$. (b) Low-magnitude TEM image showing an individual nanoflower of $W_{2}$. The HRTEM image showing the $W_{2}$ NFs with a great number of single layer and few layers. (c) The XRD patterns of commercial WS 2 (upper pattern) and WS $\mathrm{W}_{2}$ NFs (lower pattern). (d) Raman spectra of as-synthesised $W_{2}$ NFs. (e) The abundant single- and few-layered WS 2 NFs with a lattice spacing of $\sim 0.64 \mathrm{~nm}$; the greatly dispersed nanopetals offer rich active edge sites. (f) The HRTEM image of single-layer $\mathrm{WS}_{2}$. (g) and (h) The atomic structure of single layer of $\mathrm{WS}_{2}$. Reproduced from ref. 46 .

negligible amounts of Rh-B were degraded, which is evidence that the degradation occurs due to mechanical strain on the $\mathrm{WS}_{2}$ nanoflowers when subjected to ultrasonication. The degradation potential of $\mathrm{WS}_{2}$ nanoflowers was also tested on $E$. coli K12 cells. After ultrasonication for $60 \mathrm{~min},>99.99 \%$ of $E$. coli K12 cells were observed to have been destroyed. This first report on the production of singlet oxygen species and hydroxyl free radicals by $\mathrm{WS}_{2}$ nanoflowers under the conditions of ultrasonication and no light irradiation is a very significant result, and could pave the way for further application of TMDs in catalysis and as antibacterial agents.

Research into photocatalytic activity of TMDs is more common than non-light-driven catalysis, and may also be beneficial for use in antibacterial applications. Askari et al. produced porous hexagonal-phase $\mathrm{MoS}_{2}$ nanoparticles via a hydrothermal approach using sodium molybdate and thioacetamide as metal and chalcogen precursors, respectively. ${ }^{47}$ As an initial test of the nanomaterial's photocatalytic activity, different amounts of solid $\mathrm{MoS}_{2}$ nanoparticles were added to methylene blue (MB) solution and subjected to light irradiation under a $100 \mathrm{~W}$ tungsten bulb. The photocatalytic activity was highest at a $\mathrm{MoS}_{2}$ concentration of $15 \mathrm{mg} \mathrm{L}{ }^{-1}$. Once the photocatalytic capability via generation of reactive oxygen species of the

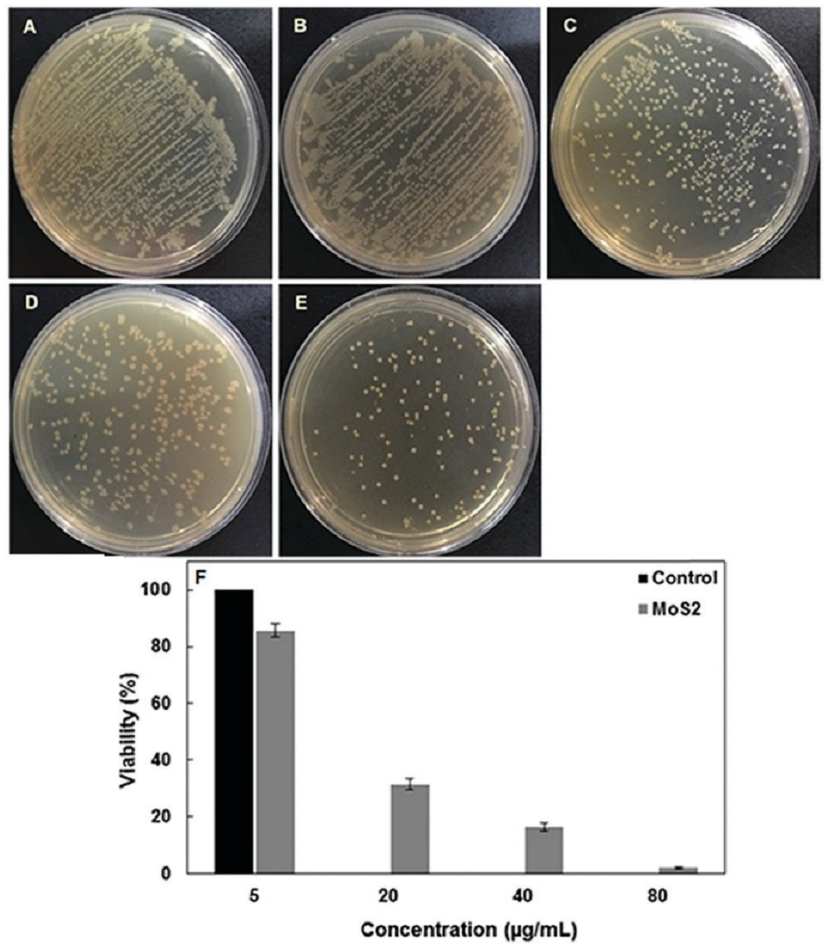

Fig. 12 Viability of E. coli cells in the absence of porous $\mathrm{MoS}_{2}(\mathrm{~A})$ and after $2 \mathrm{~h}$ exposure to different concentrations of porous $\mathrm{MoS}_{2}\left(5 \mu \mathrm{g} \mathrm{m} \mathrm{L}^{-1}(\mathrm{~B})\right.$, $20 \mu \mathrm{g} \mathrm{mL}^{-1}$ (C), $40 \mu \mathrm{g} \mathrm{mL}^{-1}$ (D) and $80 \mu \mathrm{g} \mathrm{mL}^{-1}$ (E)). (F) Cell viability is plotted as the percentage of colony-forming units of bacterial samples exposed to different concentrations of $\mathrm{MoS}_{2}$ (gray bars) relative to the control (in the absence of porous $\mathrm{MoS}_{2}$; black bar). Reproduced from ref. 47.

porous $\mathrm{MoS}_{2}$ nanoparticles was confirmed, the authors tested them for potential antibacterial applications, using both Gram positive (Staphylococcus aureus) and negative (E. coli DH5 $\alpha$ ) bacterial samples (Fig. 12). The porous $\mathrm{MoS}_{2}$ was found to be more effective as an antibacterial agent than both raw $\mathrm{MoS}_{2}$ powder and $\mathrm{MoS}_{2}$ nanosheets. ${ }^{69}$

It is clear that TMDs show huge potential for use in a variety of different biomedical applications. The monodispersity of bottom-up synthesised TMDs is hugely advantageous for use in the biomedical realm. However, more studies on the biocompatibility and in vivo toxicity of TMD nanostructures synthesised via bottom-up approaches are required to assist in the progression of this field.

\subsection{Electrocatalysis and photocatalysis}

As detailed above, many examples of the biomedical applications of 2D TMDs, in particular their antibacterial activity, are catalytic by nature. 2D TMDs have been recently explored as potential photocatalysts, in particular for use in the hydrogen evolution reaction (HER). A key factor in the reaction kinetics of the HER is the hydrogen adsorption Gibbs free energy $\left(\Delta G_{\mathrm{H}}\right){ }^{70}$ In general, the closer the value of $\Delta G_{\mathrm{H}}$ is to zero, the higher the catalytic activity. Using density functional theory, Hinnemann et al. demonstrated the potential of $\mathrm{MoS}_{2}$ as a catalyst for HER, displaying a $\Delta G_{\mathrm{H}}$ value of $0.08 \mathrm{eV}$ - very close to the ideal value of $0 \mathrm{eV}^{71}$ Nano-scale TMDs, in particular $\mathrm{MoS}_{2}$, have been 
also explored as alternatives to platinum for HER due to their low cost and high earth abundance. ${ }^{72}$ The catalytic activity of nanoscale TMDs can vary widely with phase, morphology, and defects. ${ }^{73}$ For example, the metallic phases of TMDs such as $\mathrm{MoS}_{2}$ and $\mathrm{WS}_{2}$ (1T and $1 \mathrm{~T}^{\prime}$ phases) typically exhibit higher catalytic activity in HER than their semiconducting, 2H-phase counterparts, due to the catalytic active sites being distributed on both the basal planes and the edge sites in the metallic TMD phases. $^{74}$ Thus, the preparation of TMDs for catalytic applications through bottom-up approaches can be advantageous for many reasons.

Solution-phase bottom-up synthesis allows for often more catalytically active, metastable phases (such as $1 \mathrm{~T}$ and $1 \mathrm{~T}^{\prime}$ in the case of group VI diselenides and disulfides) to be easily accessed. ${ }^{75}$ Production of TMDs exhibiting these phases via traditional methods such as $\mathrm{Li}$ intercalation and chemical exfoliation presents problems such as in non-phase pure samples, as well as metallic TMD samples often reverting back to the thermodynamically stable $2 \mathrm{H}$ phase after some time. ${ }^{76}$ However, through solution-based synthesis, involving stabilisation with appropriate ligands or surfactants, long-term stable, phase-pure $1 \mathrm{~T}$ and $1 \mathrm{~T}^{\prime}$ phases are readily accessible.

Wang et al. developed a hydrothermal approach for the synthesis of metallic $\mathrm{MoS}_{2}$ nanopetals for use as catalysts for HER. ${ }^{48} 1 \mathrm{~T}$ and $1 \mathrm{~T}^{\prime}$ phase TMDs typically exhibit higher catalytic activity because both the basal plane and edge sites are active. However, the edge sites have higher activity for HER than the basal planes, so $1 \mathrm{~T} / 1 \mathrm{~T}^{\prime}$ phase TMDs with abundant edge sites would be advantageous for HER. The petal-like $\mathrm{MoS}_{2}$ nanostructures, of an average thickness of 5 layers and a lateral size of $75 \mathrm{~nm}$, result in abundant exposed active edge sites, in turn resulting in high electrochemical activity for HER. The bottomup synthesised metallic $\mathrm{MoS}_{2}$ nanopetals showed superior HER performance compared to bulk, exfoliated and semiconducting $\mathrm{MoS}_{2}$, with an exceptionally low overpotential of $210 \mathrm{mV}$ at a current density of $10 \mathrm{~mA} \mathrm{~cm}{ }^{-2}$, and a small Tafel slope of $44 \mathrm{mV}$ per decade, approaching ideal, platinum-like behaviour (Fig. 13).

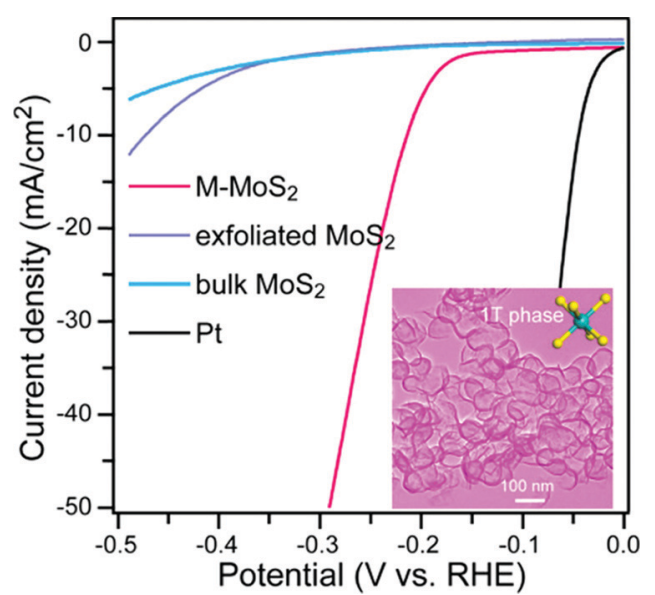

Fig. 13 Polarization curves after iR correction for $\mathrm{M}-\mathrm{MoS}_{2}$ in comparison with bulk $\mathrm{MoS}_{2}$, exfoliated $\mathrm{MoS}_{2}$ and Pt/C. Inset: TEM image of M-MoS nanopetals. Reproduced from ref. 48 .
In addition, colloidal synthesis allows for TMDs to be grown directly on a given substrate or electrode, which is highly desirable for electrocatalytic testing. Sokolikova et al. demonstrated this by growing $1 \mathrm{~T}^{\prime} \mathrm{WSe}_{2}$ nanoflowers directly on carbon paper, carbon foil and gold foil via a hot injection approach. $^{33}$ Another advantage of colloidal synthesis is the potential for controlled introduction of defects into the nanostructures, which can result in improved catalytic activity. ${ }^{77}$

Xie et al. produced defect-rich, hexagonal phase $\mathrm{MoS}_{2}$ nanosheets for application as HER catalysts via a hydrothermal approach. ${ }^{49}$ Thiourea was employed as the sulfur source, with the additional role of reducing $\mathrm{Mo}(\mathrm{vI})$ to $\mathrm{Mo}(\mathrm{Iv})$, as well defect engineering of the resultant $\mathrm{MoS}_{2}$ nanosheets. By varying the concentration of thiourea used in the hydrothermal reaction, the authors discovered that the number of defects could be controlled, thus controlling the efficacy of the $\mathrm{MoS}_{2}$ nanosheets for use as a catalyst in HER. The excess thiourea acts as a stabilising agent for the $\mathrm{MoS}_{2}$ nanosheets, partially hindering the growth, resulting in a defect-rich structure (Fig. 14). The introduction of defects significantly improves the catalytic activity as, in hexagonal/ $2 \mathrm{H}-\mathrm{MoS}_{2}$, the basal planes are inert. The defects cause cracks in the basal planes of the nanosheets, forming more edge sites, which are catalytically active. It is also worth noting that this synthesis is done on the gram-scale, and is highly scalable, which lends itself to potential industrial applications.

The photocatalytic properties of TMDs have been exploited for many applications other than HER. Removal of contaminants of natural water supplies is a huge challenge and an exciting area of research. 2D materials such as hexagonal boron nitride and TMDs are being used for this purpose, often in the form of membranes for nanofiltration. ${ }^{78}$ One such harmful contaminant is hexavalent chromium $(\mathrm{Cr}(\mathrm{vI})) .{ }^{79} \mathrm{Cr}(\mathrm{VI})$ is a known carcinogen, mutagen, as well as a toxic element to plants,

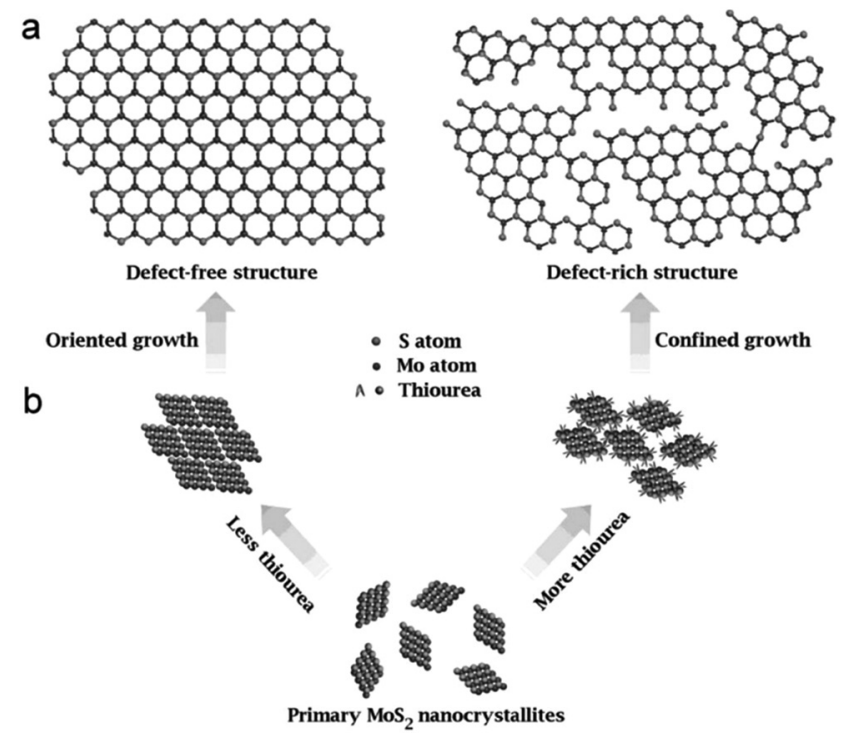

Fig. 14 (a) Structural models of defect-free and defect-rich structures. (b) As-designed synthetic pathways to obtain the above two structures. Reproduced from ref. 49. 
animals and microbes. ${ }^{80}$ However, the use of nanofiltration for removal of $\mathrm{Cr}(\mathrm{vI})$ from water supplies may have limited efficacy, as the technique suffers from drawbacks such as membrane fouling. ${ }^{81}$ An alternative route for the removal of $\mathrm{Cr}(\mathrm{VI})$ from water is the reduction of $\mathrm{Cr}(\mathrm{VI})$ to $\mathrm{Cr}$ (III), which is significantly less toxic. Chu et al. produced $2 \mathrm{H} \mathrm{MoSe}_{2}$ nanosheets via a hydrothermal route for examination of their photocatalytic activity for the reduction of $\mathrm{Cr}(\mathrm{vI})$ to $\mathrm{Cr}$ (III) under different $\mathrm{pH}$ conditions. ${ }^{50}$ An advantage of $\mathrm{MoSe}_{2}$ over other photocatalysts is that it exhibits photocatalytic activity over the UV, visible and IR regions, whereas many other photocatalysts only operate in the UV and visible regions, which account for only $46 \%$ of the solar energy reaching earth. The $\mathrm{MoSe}_{2}$ prepared at a $\mathrm{pH}$ of 2 had higher absorption and a larger specific area, resulting in the best photocatalytic activity. After $3 \mathrm{~h}, \mathrm{MoSe}_{2}$ resulted in $99 \%, 91 \%$ and $98 \%$ reduction of $\operatorname{Cr}(\mathrm{VI})$ to $\operatorname{Cr}(\mathrm{III})$ under $\mathrm{UV}$, visible and IR light irradiation, respectively. The use of "bottom-up" synthetic methods in this case allowed for the production of $\mathrm{MoSe}_{2}$ with ultrahigh specific surface area, which aided in the exceptional performance of the photocatalyst.

\subsection{Electronics and optoelectronics}

2D materials have been the subject of much research interest for potential electronic applications in recent years. For some time, graphene was the main focus, due to its high electron mobility, flexibility, transparency, tensile strength and thermal conductivity. ${ }^{82}$ However, the major disadvantage of graphene is that it lacks an electronic bandgap, thus limiting its potential application as a material for use in transistors. ${ }^{83}$ 2D TMDs also exhibit many of the advantageous properties of graphene, with the additional benefit of having a non-zero bandgap, as well as exhibiting phase-dependent conductivity behaviour, ranging from metallic (e.g. $1 \mathrm{~T} \mathrm{MoS}_{2}$ ) to semiconducting (e.g. $2 \mathrm{H} \mathrm{MoS}$ ). ${ }^{84}$

A significant drawback of solution-phase exfoliated 2D materials is their polydispersity. ${ }^{85}$ For electronic device applications, uniform sheets of material are required, to ensure consistency across devices if produced on a large scale. ${ }^{86,87}$ This can be achieved by synthetic methods such as hydro/solvothermal and hot-injection approaches; however the photophysics of colloidal TMDs are not well studied. Schiettecatte et al. produced ultrathin, 2-4-layer $\mathrm{MoS}_{2}$ nanosheets via hot-injection. ${ }^{58}$ Using transient absorption spectroscopy, a detailed study was carried out on the charge carrier dynamics in colloidal $\mathrm{MoS}_{2}$. The authors found that the carrier dynamics in colloidal $\mathrm{MoS}_{2}$ followed a very similar behaviour to that of CVD-grown $\mathrm{MoS}_{2}$, suggesting that colloidal synthesis of $\mathrm{MoS}_{2}$ yields nanosheets of comparable quality to those grown by state-of-the-art CVD.

Similarly, Zhou et al. produced ultrathin $2 \mathrm{H} \mathrm{WS}_{2}$ via a hotinjection approach to gain insight into the photophysics of the nanosheets necessary to exploit their potential application in optoelectronics. ${ }^{36}$ They too found that the colloidal $\mathrm{WS}_{2}$ nanosheets exhibited a very similar photophysical behaviour to that of CVD-grown $\mathrm{WS}_{2}$. This demonstrates the huge potential of bottom-up synthesis of TMDs, as it is possible to produce CVD-quality nanosheets at a fraction of the cost and under much less extreme conditions.
One of the advantages of using colloidal suspensions of TMDs in electronics is that they can be spin-coated or printed onto suitable substrates, to easily and inexpensively prepare TMD thin films. ${ }^{88,89}$ As mentioned previously, Mastria et al. developed a shape-transformation approach involving hotinjection, to produce ultrathin, oleylamine-stabilised, colloidal $\mathrm{WS}_{2}$ nanosheets for the production of solution processable, conducting thin films for device integration. ${ }^{41}$ Treatment of the thin films after deposition with diluted superacid resulted in partial removal of the oleylamine ligands, helping the nanosheets to orient parallel to the substrate, which is critical for increasing the planar conductivity of the $\mathrm{WS}_{2}$ thin-film. The 40-140 $\mu \mathrm{m}$ thick films, before post-deposition treatment, were shown to be highly insulating, due to the presence of oleylamine ligands. However, after treatment and partial removal of oleylamine, the sheet resistivity was dramatically reduced, displaying conductivity values comparable to that of pristine few-layer to monolayer semiconducting $\mathrm{WS}_{2}$ nanosheets obtained by mechanical exfoliation. ${ }^{90}$

Son et al. developed a gram-scale hot-injection synthesis for the production of uniform, monodisperse, 2-layer-thick $\mathrm{MoS}_{2} \cdot{ }^{57}$ Using HRTEM and STEM, the authors were able to show that the colloidal $\mathrm{MoS}_{2}$ was thinner and significantly more monodisperse than exfoliated $\mathrm{MoS}_{2}$. A resistive random-access memory (RRAM) device was prepared using the colloidal $\mathrm{MoS}_{2}$ via a spray coating deposition technique on aluminium electrodes. A similar device using exfoliated $\mathrm{MoS}_{2}$ was also fabricated as a control. The synthetic $\mathrm{MoS}_{2}$ RRAM exhibited superior uniform device performance, displaying an on/off ratio 10000 times higher than that of the exfoliated $\mathrm{MoS}_{2}$-based RRAM device, due in part to the uniformity of the colloidal $\mathrm{MoS}_{2}$ nanosheets. By conducting strain analysis of colloidal $\mathrm{MoS}_{2}$-based RREM using a flexible substrate, the authors demonstrated the exciting potential of colloidal $\mathrm{MoS}_{2}$ for use in wearable, flexible electronics (Fig. 15).

Another emerging application of TMD nanostructures is as field emitters. Field emission involves the emission of electrons from a material induced by an electric field. ${ }^{91}$ Generally, metallic and semiconducting nanomaterials are good natural field emitters, particularly 1D and 2D nanostructures, which can cause local electric field enhancement due to their high aspect ratio. ${ }^{92}$ However, the field emission effects from more exotic morphologies of TMDs such as nanoflowers are not well understood. Giubileo et al. produced $\mathrm{MoS}_{2}$ via a hydrothermal approach to investigate their field emission characteristics. ${ }^{52}$ The $2 \mathrm{H}$ phase, 100-200 nm diameter nanoflowers were probed using tungsten tips as electrodes inside the SEM chamber. The use of small tips as electrodes allowed the field emission to be probed locally, at areas as small as $1 \mu^{2}$, showing that the protruding sheets of the nanoflowers acted as strong field emitters, with electronic emission occurring at electric fields as low as $12 \mathrm{~V} \mu \mathrm{m}^{-1}$. These field emitting $\mathrm{MoS}_{2}$ nanoflowers could find application in areas such as cathode materials for electron microscopy and optical displays, to name a few. ${ }^{93-95} \mathrm{It}$ is important to mention that this unique field emission behaviour is characteristic of the flower-like morphology obtained 


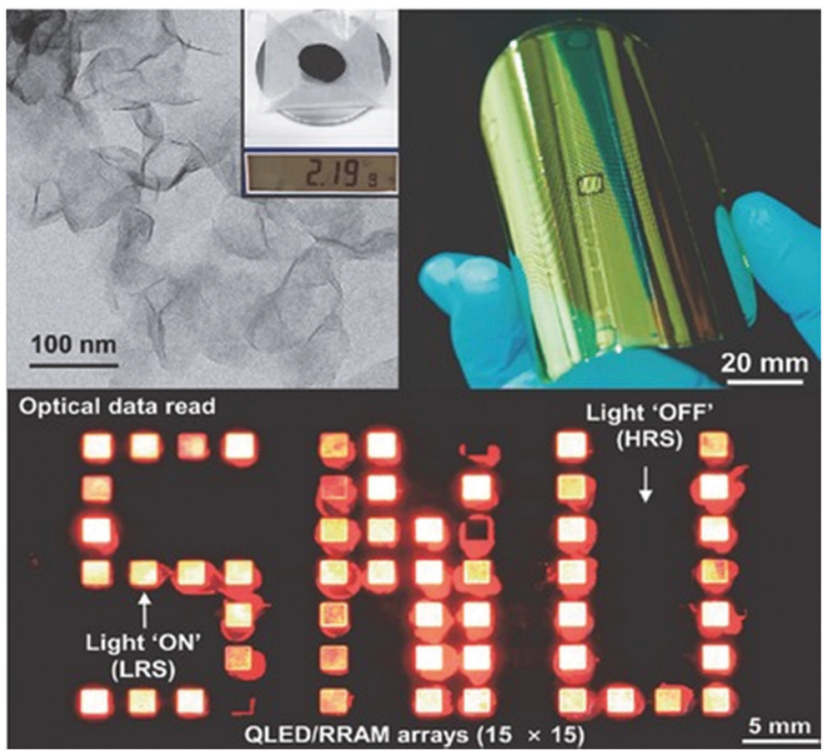

Fig. 15 (top-left) TEM image of 2-layer thick $\mathrm{MoS}_{2}$ nanosheets. The inset shows that the synthesis occurs on a gram scale. (top-right) Flexible RREM device made from $\mathrm{MoS}_{2}$ nanosheets via a spray deposition technique (bottom) QLED-RRAM array based on $\mathrm{MoS}_{2}$ nanosheets and CdSe QDs. Reproduced from ref. 57.

via hydrothermal synthesis, highlighting the advantages of bottom-up synthesis and interesting applications which can arise from these synthetic approaches.

An emerging potential application for 2D TMD-based nanomaterials is valleytronics, which involves the use of the electron wave quantum number in a crystalline material to encode data by controlling the photon angular momentum (circular polarisation state) via circular polarised light. ${ }^{96}$ This area is rapidly developing and so far is mostly focused on the use of atomic layered transition metal dichalcogenides (TMDs), such as $\operatorname{MoS}_{2}{ }^{97}$ Hexagonal TMDs are of particular interest for valleytronics applications as they lack inversion symmetry, which allows for different circular dichroism (CD) behaviour in each of the valleys. ${ }^{98}$ The induction of chirality in $2 \mathrm{D}$ materials may be of particular interest for valleytronics applications, due to the strong interaction with circularly polarized light. This has recently been achieved in $\mathrm{MoS}_{2}$ by liquid-phase exfoliation in the presence of chiral ligands such as cysteine and penicillamine. ${ }^{99}$ The functionalized $\mathrm{MoS}_{2}$ showed multiple intense $\mathrm{CD}$ peaks, far beyond the onset of $\mathrm{CD}$ signals due to the ligands alone (Fig. 16). The benefit of bottom-up synthetic approaches is that they allow for the production of high quality, size-controlled samples, with the ability to functionalise the nanomaterial in situ. Thus, the development of these synthetic techniques has the potential to mass produce monolayer-rich dispersions of colloidal, chiral TMDs for use in valleytronics applications.

\subsection{Sensing applications}

Gas sensors are commonplace in both industrial and residential settings, and are vital to protect people from potential exposure to harmful, noxious gases, as well as having application in medical diagnostics. ${ }^{100}$ In recent years however,
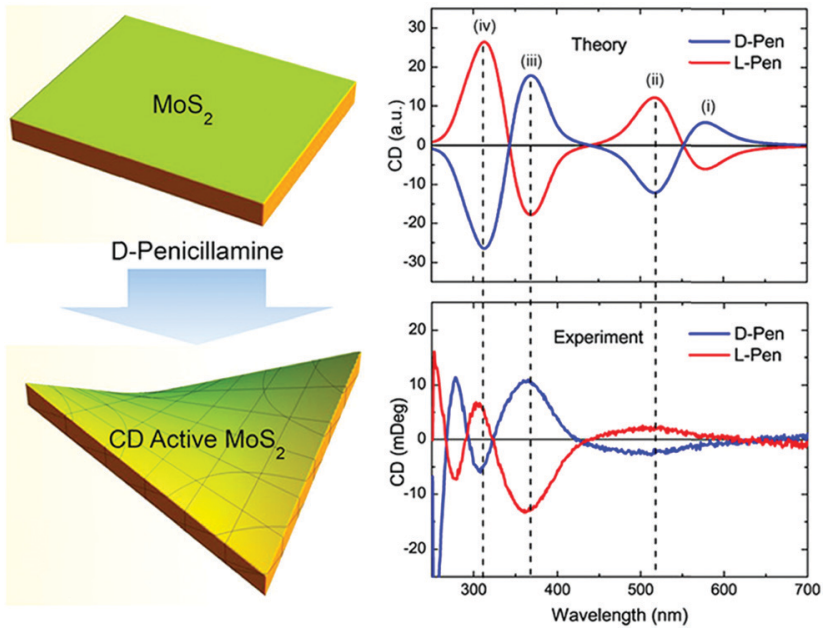

Fig. 16 (left) Schematic showing functionalisation of $\mathrm{MoS}_{2}$ with penicillamine and subsequent induction of chirality. (right) Theoretical (top) and experimental (bottom) CD spectra for L- and D-penicillamine functionalised $\mathrm{MoS}_{2}$. Reproduced from ref. 99.

there has been significant demand for, and interest in, wearable electronics, and integration of gas sensors into the likes of smartphones and smart watches, with the aim of providing the user with real-time information about their environment, as well as to monitor their health and wellbeing. ${ }^{101,102} 2 \mathrm{D}$ materials have been explored for small scale gas sensors for device integration as their large surface to volume ratio means there are abundant sites available for target gases to bind to, allowing for exceptional sensitivity. ${ }^{103}$ Furthermore, TMDs in particular are regarded as good candidates for gas sensing technologies, as they can operate at room temperature due to their high electron mobility, which significantly reduces power consumption. ${ }^{104}$

Solution-based bottom-up approaches for the synthesis of TMDs for gas sensing technologies are preferable, as they allow for a high yield of the product, at low-cost and with good controllability over particle size and morphology. Thang et al. synthesised a $\mathrm{MoS}_{2}$-based gas sensor, fabricated by drop-casting $\mathrm{MoS}_{2}$ nanoflowers - obtained via a hydrothermal approach - on platinum electrodes. ${ }^{53}$ The sensor, which operated at room temperature, showed excellent selectivity to sensing $\mathrm{NO}_{2}$ gas in the presence of other interfering gases and an exceptionally low detection limit of 84 parts per billion, and the sensing properties did not vary with environmental humidity. It is also worth noting that the authors attributed the exceptional sensing capabilities of this $\mathrm{MoS}_{2}$-based $\mathrm{NO}_{2}$ sensor to the thin petals of the flower-like morphology obtained via hydrothermal synthesis.

TMD-based nanomaterials are also good candidates for solution-phase chemical sensing for a variety of applications, from environmental (e.g. heavy metal ion detection) to medical diagnostics. ${ }^{105,106}$ Both monolayer TMD nanosheets and TMD QDs are luminescent in the visible region, making them viable candidates for use in fluorescent sensors. Sun $e t$ al. synthesised $\mathrm{MoS}_{2}$ QDs via a hydrothermal approach for the detection of rutin, a glycoside found in fruits and grains which has 


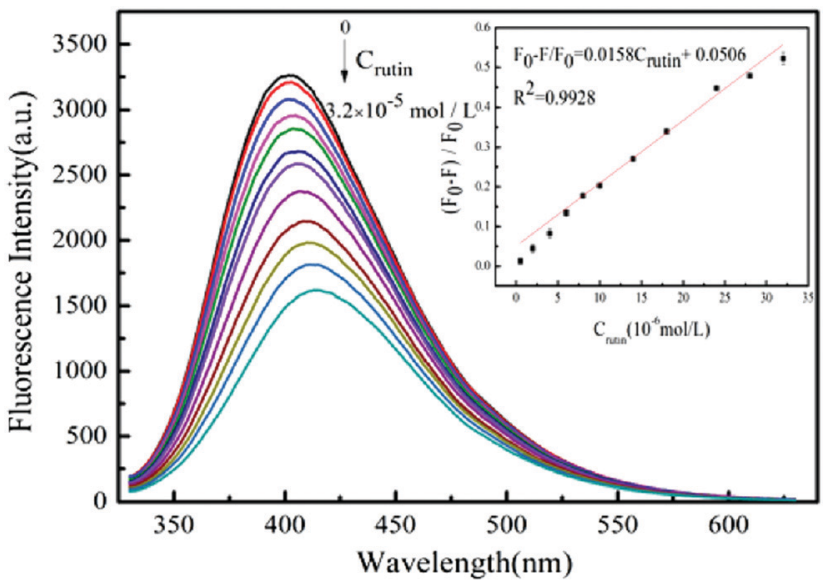

Fig. 17 Fluorescence spectra of $\mathrm{MoS}_{2}$ quantum dots (QDs) with various concentrations of rutin. The inset shows the linear relationship between $F_{0}-F / F_{0}$ and rutin concentration. Reproduced from ref. 54.

been shown to have medicinal properties. ${ }^{54,107}$ The authors found that on contact of rutin with $\mathrm{MoS}_{2}$ QDs, the fluorescence intensity decreased, and this decrease became more pronounced on addition of higher concentrations of rutin (Fig. 17). The quenching mechanism was determined to be due to the inner filter effect.

The $\mathrm{MoS}_{2}$ QD fluorescent sensor was found to be comparable to other, more complicated and expensive detection techniques such as high-performance liquid chromatography (HPLC) and microsequential injection analysis (MIA), with a lower detection limit of $0.35 \mu \mathrm{M}$. The sensor also showed high selectivity towards detection of rutin in the presence of metal ions and common pharmaceutical additives.

\subsection{Energy harvesting and storage}

As previously mentioned, as the demand for wearable electronics has soared in recent years, so too has the demand for energy harvesting materials for integration into these devices as a green, sustainable energy source. Harvesting energy created by the body through movement and heat and converting it to electricity is an area of significant interest. ${ }^{108}$ One such energy harvesting device is the thermoelectric generator, which generates electricity from a temperature gradient across a material. For a thermoelectric generator to be highly efficient, the material needs to have a high Seebeck coefficient, high electrical conductivity, and low thermal conductivity. ${ }^{109}$ Xie et al. developed a thermoelectric nanogenerator based on a $2 \mathrm{H}-\mathrm{MoS}_{2} /$ graphene hybrid material via a hydrothermal approach. ${ }^{110}$ The reason for choosing a hybrid material is because both graphene and $\mathrm{MoS}_{2}$ have advantages and drawbacks for use in a thermoelectric generator. $\mathrm{MoS}_{2}$ has a high Seebeck coefficient and low thermal conductivity; however its large bandgap results in low electrical conductivity. ${ }^{111}$ Graphene, on the other hand, is a zero-bandgap semimetal, so has high electrical conductivity, but suffers from high thermal conductivity and a low Seebeck coefficient. The resulting $\mathrm{MoS}_{2} /$ graphene nanocomposite was found to have improved electrical conductivity compared to pristine $\mathrm{MoS}_{2}$, while still maintaining low thermal conductivity and a high Seebeck coefficient. A flexible thermoelectric generator was produced using this $\mathrm{MoS}_{2}$ /graphene nanocomposite, and was shown to generate an output voltage 2 times higher than that generated by pure $\mathrm{MoS}_{2}$ and 8 times higher than that generated by pure graphene thermoelectric nanogenerators.

Another promising application of TMD nanomaterials is their use as anode materials in lithium ion batteries. A huge advantage of bottom-up synthesis of TMDs is that it can offer great controllability over properties such as size, phase and morphology, which can be crucial for energy storage applications. Lu et al. prepared $\mathrm{MoS}_{2}$ nanoflowers via a hydrothermal approach, using the reaction temperature to tune the interlayer distance between the constituent $\mathrm{MoS}_{2}$ nanosheets. ${ }^{51}$ It was shown that at higher temperatures, the interlayer spacing between the $\mathrm{MoS}_{2}$ nanosheets was decreased, as evidenced by the shift to higher angles of the (002) peak in the XRD patterns of different $\mathrm{MoS}_{2}$ samples. The increased reaction temperature also resulted in the formation of thinner nanosheets and nanoflowers with increased surface area, which is desirable for optimised lithium ion insertion-extraction. The sample with the best electrochemical activity was synthesised at $200{ }^{\circ} \mathrm{C}$, exhibiting superior rate capability and lithium storage capacity. At this temperature, the $\mathrm{MoS}_{2}$ nanoflowers were found to have an optimal interlayer distance $(0.65 \mathrm{~nm})$ as well as improved crystallinity, the presence of some defects, and an enhanced specific surface area, which are also vital for optimising the electrochemical performance.

\subsection{Photonics applications}

Saturable absorption is a phenomenon by which the absorption of light by a given material decreases with increasing light intensity. The saturable absorption properties of 2D materials have been extensively studied in recent years for Q-switching and mode-locking in ultrafast compact pulsed lasers. ${ }^{112}$ Few layer to monolayer TMDs such as $\mathrm{MoS}_{2}$ and $\mathrm{WS}_{2}$ are particularly useful for these applications as their direct bandgap falls in the visible range, whereas many other $2 \mathrm{D}$ materials used for these purposes are limited to operation in the infrared region. ${ }^{113,114}$ Few-layer to monolayer nanosheets are often studied for their saturable absorption properties; however more exotic morphologies such as nanoflowers obtained through bottom-up synthesis are neglected. Sun et al. investigated the saturable absorption properties of $\mathrm{MoS}_{2}$ nanosheets and, for the first time, nanoflowers synthesised via a hydrothermal approach. ${ }^{55}$ The colloidal $\mathrm{MoS}_{2}$ samples were then mixed with $1 \%$ polymethyl methacrylate (PMMA) in toluene solution and spin-coated onto a quartz substrate to fabricate the saturable absorber (SA) for insertion into the Yb:GAB laser cavity (Fig. 18).

The nanosheets had Q-switching properties comparable to those of other $\mathrm{MoS}_{2}$ SAs obtained via exfoliation, displaying shortest pulse widths of 281 and 216 ns for single and dual wavelength laser sources, respectively. The nanoflowers, however, displayed superior optical switching behaviour compared to the nanosheets, with pulse widths of 209 and 198 ns for single 


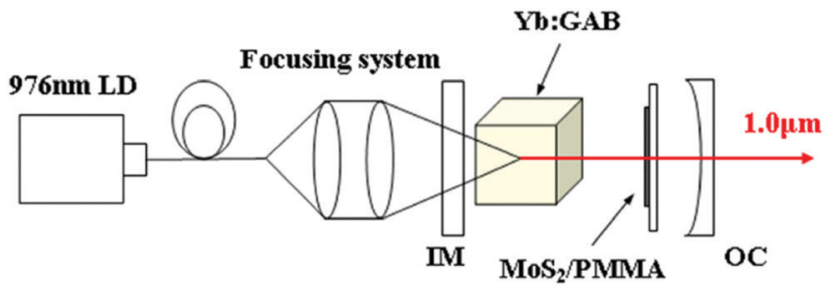

Fig. 18 Schematic experimental setup of the $\mathrm{Q}$-switched $\mathrm{Yb}^{3+}$ :GAB solidstate laser. Reproduced from ref. 55.

and dual wavelengths. The authors attributed this enhanced non-linear optical behaviour to the unique morphology of the nanoflowers obtained via hydrothermal synthesis. Thus, further development and research in the field of bottom-up synthesis of TMDs may open the door for large-scale application of TMDs in lasers, due to its cost efficiency, scalability, and high-quality products.

Group VI monolayer 2H TMDs such as $\mathrm{MoS}_{2}$ and $\mathrm{WS}_{2}$ display photoluminescence due to the indirect to direct bandgap transition on being reduced from the bulk to the monolayer. This phenomenon has led to a multitude of potential applications of monolayer TMDs in photonics and optoelectronics. One of the limitations of the use of monolayer TMDs in photonics applications is the quantum yield (QY). The QYs of many pristine monolayer TMDs are often very low $(<1 \%)$, likely due to non-radiative electron-hole recombination mediated by defects. ${ }^{115,116}$ However, chemical doping is an effective method of passivation of these non-radiative decay pathways, resulting in significant enhancement of the QY of monolayer TMDs. Mouri et al. discovered that doping with 2,3,5,6-tetrafluoro7,7,8,8-tetracyanoquinodimethane (F4TCNQ) and 7,7,8,8tetracyanoquinodimethane (TCNQ), both p-type dopants, dramatically increased the photoluminescence (PL) quantum yield of mechanically exfoliated monolayer $\operatorname{MoS}_{2} \cdot{ }^{117}$ Amani et al. reported similar findings upon the treatment of monolayer $\mathrm{MoS}_{2}$ with bis(trifluoromethane)sulfonimide (TFSI), an organic superacid, resulting in a PLQY enhancement from $0.6 \%$ to $>95 \%{ }^{118}$ Solution-based "bottom-up" synthetic methods allow for the doping of TMDs in situ, which may pave the way for the production of size-controlled, high PLQY monolayer TMDs in a one-pot process. Highly luminescent monolayer TMDs synthesised via bottom-up approaches may find application in areas of photonics such as highly efficient LEDs, as single photon emitters for quantum computing, and in circularly polarised light emission devices, to name a few. ${ }^{119-121}$

\subsection{Membranes and nanofiltration applications}

2D nanomaterials of atomic thickness are excellent nano-building blocks to develop high-performance separation membranes, as these nanomaterials exhibit extraordinary permeation behaviour. This opens a new avenue to ultra-fast and highly selective laminar membranes with tailored functions and properties for nanofiltration and purification. ${ }^{122}$ So far, most of the research in this area has been focused on graphene- and graphene oxide-based membranes, but atomic layered TMDs such as $\mathrm{MoS}_{2}$ and $\mathrm{WS}_{2}$ have also attracted some attention. ${ }^{123-131}$ For example, it was

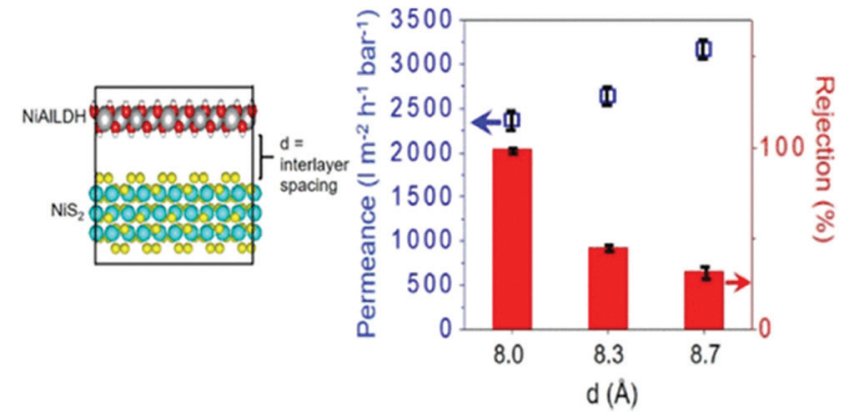

Fig. 19 Left: Schematic presentation of control of the interlayer spacing of anionic TMD laminates using cationic layered double hydroxide (LDH) nanosheets. Right: Permeance and rejection of methyl orange $\mathrm{MO}$ ) dye in acetone through $250 \pm 20 \mathrm{~nm}$ thick $\mathrm{NiS}_{2} / \mathrm{NiAlLDH}$ membranes as a function of the interlayer distance. Reproduced from ref. 130.

demonstrated that $\mathrm{MoS}_{2^{-}}$and $\mathrm{WS}_{2}$-based membranes exhibit water permeance 2- to 5-fold greater than that of graphene oxide-based membranes of comparable thickness. ${ }^{123,124}$

Sun et al. used vacuum filtration of an exfoliated $\mathrm{MoS}_{2}$ solution through a polycarbonate membrane to produce new membranes showing much higher water permeance than previously described graphene membranes, with similar thickness and rejection ratio due to the higher level of interaction between the $\mathrm{MoS}_{2}$ sheets. ${ }^{124}$ In addition, it was found that the nanochannels in the $\mathrm{MoS}_{2}$ membranes did not deform under pressures as high as $1.0 \mathrm{MPa}$, as has been shown by the linear relationship between pressure and water permeance. Furthermore, this type of membrane demonstrated high chemical stability even under harsh conditions, and therefore can be employed repeatedly.

Thus, TMD-based membranes are very promising for potential nanofiltration of organic compounds and water purification applications. However, a great challenge lies in the precise control of the interlayer spacing and corresponding membrane porosity and selectivity. An interesting approach to address some of these issues was recently reported by Ang and Chew. ${ }^{130}$ These researchers prepared a new type of membrane by stacking anionic TMD nanosheets, consisting of $\mathrm{MS}_{2}(\mathrm{M}=\mathrm{Ni}, \mathrm{Mo}$, and $\mathrm{W})$, and cationic layered double hydroxide (LDH) nanosheets (Fig. 19). This method enabled them to achieve precise control of the interlayer spacing of anionic TMD laminates, resulting in TMD/LDH lamellar membranes which exhibited almost 100\% rejection of selected organic dyes dissolved in acetone, while maintaining excellent long-term stability at an ultrafast permeance that is $2-3$ orders-ofmagnitude higher than that of other reported membranes with similar rejection.

This proposed hetero-assembly strategy using oppositely charged nanosheets is a very promising method to produce inorganic 2D-based membranes with a controllable interlayer distance for other gas or liquid separation applications.

\section{Conclusions and future outlook}

In this review, we have considered the various methods of solution-based bottom-up synthesis of TMDs and the properties 
of the resulting products. It was shown that these techniques enable the production of TMDs of varying shapes, sizes, morphologies, and phases, and we also gave some insight into how the variation of reaction conditions can affect these properties. It is clear that, if developed sufficiently, these synthetic methods may combine the cost-effectiveness and scalability of techniques such as LPE with the enhanced level of controllability provided by CVD, often at a fraction of the cost. The unique morphologies and phases that are readily obtained via these bottom-up synthetic regimes give rise to exciting applications as photocatalysts, anti-cancer and antibacterial agents, as well as for integration into electronics devices.

The high quality and narrow size distribution of synthetic colloidal TMD-based nanomaterials, in combination with their unique physical and chemical properties, has resulted in significant advancements in the development of applications for these materials in recent years. It was demonstrated that 2D TMD materials are very promising for a range of biomedical applications including biosensing, drug delivery and photodynamic/photothermal therapy. However, despite the vast amount of progress made in recent years, and the obvious potential of these nanomaterials, they remain of academic interest only. One of the main concerns is the unknown potential toxicity of 2D nanostructures, which could limit their in vivo biomedical applications. The toxicity of 2D nanomaterials is still poorly studied and understood, and much detailed research on the biocompatibility and toxicity of TMD nanostructures is vital for their further advancement and use in biomedical applications.

TMDs possess unique catalytic properties which have been exploited in electro- and photocatalysis, in particular in the hydrogen evolution reaction (HER) and photo-oxidation of organic materials. However, further detailed experimental and theoretical studies are necessary to fully understand all phenomena, and to develop new, more efficient and selective catalysts, which will be crucial for the progression of relevant green chemistry sectors.

The 2D morphology and unique properties of TMDs such as semiconductor state, conductivity, flexibility, transparency and large surface area make these materials highly promising for future development of new wearable, flexible electronic devices. The promotion of synthetic colloidal TMD nanomaterials is especially important for these applications as they can be easily processed by standard solution and printing techniques, enabling new, cost-effective, flexible electronic devices.

2D TMD nanomaterials can also be tailored to form either fluorescent emitters or efficient fluorescence quenchers, making them powerful platforms for fabricating a series of optical sensors and biosensors to detect various targets, including gases, metal ions, biomolecules and potentially viral species. Despite the significant recent progress, TMD-based sensors still require further improvements in terms of detection limits and selectivity. The colloidal chemistry synthetic approaches offer excellent opportunities in this area, enabling the production of monolayers of photoluminescent TMD materials, which can be easily functionalised by standard wet chemistry techniques for targeted sensing applications. The photoluminescence properties of monolayered colloidal TMDs are also important for photonics applications, particularly in light emitting and laser devices.

TMD nanomaterials have emerging applications in energy harvesting and storage devices. The use of colloidal $\mathrm{MoS}_{2}$ and related materials as anodes in lithium ion batteries is particularly important. The bottom-up synthesis of TMDs enables the achievement of necessary control over size, phase and morphology of these nanomaterials and allows us to use efficient screen-printing techniques with colloidal inks, which are required for cost-effective production of batteries.

Colloidal synthetic 2D TMD nanomaterials are excellent materials for production of laminar membranes which are capable of providing exceptional performance and separation, even at very low membrane thickness. It has been shown that TMD-based membranes can outperform similar graphene oxide-based membranes of comparable thickness. The porosity of these membranes can be controlled and tuned by the dimensions of TMD flakes and by the addition of various interstitial nanoparticles or nanowires. In addition, these membrane materials can also be easily functionalised with appropriate groups to provide the necessary level of selectivity for targeted rejection and separation of mixtures of products. Thus, TMD-based membranes are expected to offer a number of advantages and their further development deserves significant attention.

Finally, the development of new chiral 2D TMD nanomaterials is expected to open up a vast range of new potential applications in valleytronics, spintronics, chiral sensing and asymmetric photocatalysis. However, the area of chiral 2D nanomaterials and their applications are minimally explored to date and this field is still in its infancy. Colloidal 2D TMD materials are excellent candidates for this research as chirality in these nanostructures can be induced by a simple synthesis in the presence of chiral ligands, or by the solution exchange of achiral ligands with appropriate enantiomeric molecules. Since chirality plays a crucial role in chemical and biological recognition and defines many interactions between chemical and biological species, these nanomaterials are also expected to have a significant impact on the fast-developing nanobiotechnology and nanomedicine disciplines.

\section{Conflicts of interest}

There are no conflicts to declare.

\section{Acknowledgements}

The authors gratefully thank the Irish Research Council (projects: GOIPG/2019/2788) and the SFI Bioeconomy Research Centre, Biorbic (project: SFI 16/RC/3889) for financial support. 


\section{References}

1 K. S. Novoselov, A. K. Geim, S. v. Morozov, D. Jiang, Y. Zhang, S. v. Dubonos, I. v. Grigorieva and A. A. Firsov, Science, 2004, 306, 666-669.

2 Y. Hernandez, V. Nicolosi, M. Lotya, F. M. Blighe, Z. Sun, S. De, I. T. McGovern, B. Holland, M. Byrne, Y. K. Gun'ko, J. J. Boland, P. Niraj, G. Duesberg, S. Krishnamurthy, R. Goodhue, J. Hutchison, V. Scardaci, A. C. Ferrari and J. N. Coleman, Nat. Nanotechnol., 2008, 3, 563-568.

3 J. N. Coleman, M. Lotya, A. O’Neill, S. D. Bergin, P. J. King, U. Khan, K. Young, A. Gaucher, S. De, R. J. Smith, I. v. Shvets, S. K. Arora, G. Stanton, H. Y. Kim, K. Lee, G. T. Kim, G. S. Duesberg, T. Hallam, J. J. Boland, J. J. Wang, J. F. Donegan, J. C. Grunlan, G. Moriarty, A. Shmeliov, R. J. Nicholls, J. M. Perkins, E. M. Grieveson, K. Theuwissen, D. W. McComb, P. D. Nellist and V. Nicolosi, Science, 2011, 331, 568-571.

4 C. Ataca, H. Şahin and S. Ciraci, J. Phys. Chem. C, 2012, 116, 8983-8999.

5 K. F. Mak, C. Lee, J. Hone, J. Shan and T. F. Heinz, Phys. Rev. Lett., 2010, 105, 136805.

6 A. Splendiani, L. Sun, Y. Zhang, T. Li, J. Kim, C.-Y. Chim, G. Galli and F. Wang, Nano Lett., 2010, 10, 1271-1275.

7 H. Tian, J. Tice, R. Fei, V. Tran, X. Yan, L. Yang and H. Wang, Nano Today, 2016, 11, 763-777.

8 A. Soni, C. M. Pandey, M. K. Pandey and G. Sumana, Anal. Chim. Acta, 2019, 1055, 26-35.

9 Y. Xu, H. Cao, Y. Xue, B. Li and W. Cai, Nanomaterials, 2018, 8, 942.

10 M. Bodík, A. Annušová, J. Hagara, M. Mičušík, M. Omastová, M. Kotlár, J. Chlpík, J. Cirák, H. Švajdlenková, M. Anguš, A. M. Roldán, P. Veis, M. Jergel, E. Majkova and P. Šiffalovič, Phys. Chem. Chem. Phys., 2019, 21, 12396-12405.

11 C. Backes, B. M. Szydłowska, A. Harvey, S. Yuan, V. VegaMayoral, B. R. Davies, P. L. Zhao, D. Hanlon, E. J. G. Santos, M. I. Katsnelson, W. J. Blau, C. Gadermaier and J. N. Coleman, ACS Nano, 2016, 10, 1589-1601.

12 Q. Fu, W. Wang, L. Yang, J. Huang, J. Zhang and B. Xiang, RSC Adv., 2015, 5, 15795-15799.

13 M. H. Khan, H. K. Liu, X. Sun, Y. Yamauchi, Y. Bando, D. Golberg and Z. Huang, Mater. Today, 2017, 20, 611-628.

14 W. Liu, H. Li, C. Xu, Y. Khatami and K. Banerjee, Carbon, 2011, 49, 4122-4130.

15 J. A. Wilson and A. D. Yoffe, Adv. Phys., 1969, 18, 193-335.

16 G. W. Morey and P. Niggli, J. Am. Chem. Soc., 1913, 35, 1086-1130.

17 T. Dubois and G. Demazeau, Mater. Lett., 1994, 19, 38-47.

18 J. H. Zhan, Z. D. Zhang, X. F. Qian, C. Wang, Y. Xie and Y. T. Qian, J. Solid State Chem., 1998, 141, 270-273.

19 R. Fan, X. Chen and Z. Chen, Chem. Lett., 2000, 920-921.

20 H. A. Therese, J. Li, U. Kolb and W. Tremel, Solid State Sci., 2005, 7, 67-72.

21 D. Chakravarty and D. J. Late, RSC Adv., 2015, 5, 21700-21709.

22 Y. Liu, N. Zhang, H. Kang, M. Shang, L. Jiao and J. Chen, Chem. - Eur. J., 2015, 21, 11878-11884.
23 P. P. Wang, H. Sun, Y. Ji, W. Li and X. Wang, Adv. Mater., 2014, 26, 964-969.

24 V. K. Lamer and R. H. Dinegar, J. Am. Chem. Soc., 1950, 72, 4847-4854.

25 C. B. Murray, D. J. Norris and M. G. Bawendi, J. Am. Chem. Soc., 1993, 115, 8706-8715.

26 X. Bai, F. Purcell-Milton and Y. Gun'ko, Nanomaterials, 2019, 9, 85.

27 C. B. Murray, C. R. Kagan and M. G. Bawendi, Annu. Rev. Mater. Sci., 2000, 30, 545-610.

28 A. Ghorai, A. Midya and S. K. Ray, New J. Chem., 2018, 42, 3609-3613.

29 A. Bayat and E. Saievar-Iranizad, J. Lumin., 2017, 185, 236-240.

30 L. Zhou, S. Yan, H. Wu, H. Song and Y. Shi, Catalysts, 2017, 7, 18.

31 A. Ghorai, S. Bayan, N. Gogurla, A. Midya and S. K. Ray, ACS Appl. Mater. Interfaces, 2017, 9, 558-565.

32 W. Yin, X. Bai, P. Chen, X. Zhang, L. Su, C. Ji, H. Gao, H. Song and W. W. Yu, ACS Appl. Mater. Interfaces, 2018, 10, 43824-43830.

33 M. S. Sokolikova, P. C. Sherrell, P. Palczynski, V. L. Bemmer and C. Mattevi, Nat. Commun., 2019, 10, 1-8.

34 N. Savjani, E. A. Lewis, M. A. Bissett, J. R. Brent, R. A. W. Dryfe, S. J. Haigh and P. O'Brien, Chem. Mater., 2016, 28, 657-664.

35 W. Jung, S. Lee, D. Yoo, S. Jeong, P. Miró, A. Kuc, T. Heine and J. Cheon, J. Am. Chem. Soc., 2015, 137, 7266-7269.

36 P. Zhou, I. Tanghe, P. Schiettecatte, D. van Thourhout, Z. Hens and P. Geiregat, J. Chem. Phys., 2019, 151, 164701.

37 W. Zhao, Z. Ghorannevis, K. K. Amara, J. R. Pang, M. Toh, X. Zhang, C. Kloc, P. H. Tan and G. Eda, Nanoscale, 2013, 5, 9677-9683.

38 A. Berkdemir, H. R. Gutiérrez, A. R. Botello-Méndez, N. Perea-López, A. L. Elías, C. I. Chia, B. Wang, V. H. Crespi, F. López-Urías, J. C. Charlier, H. Terrones and M. Terrones, Sci. Rep., 2013, 3, 1-8.

39 J. Q. Geisenhoff, A. K. Tamura and A. M. Schimpf, Chem. Commun., 2019, 55, 8856-8859.

40 J. W. Seo, Y. W. Jun, S. W. Park, H. Nah, T. Moon, B. Park, J. G. Kim, Y. J. Kim and J. Cheon, Angew. Chem., Int. Ed., 2007, 46, 8828-8831.

41 R. Mastria, R. Scarfiello, D. Altamura, C. Giannini, A. Liscio, A. Kovtun, G. V. Bianco, G. Bruno, V. Grillo, A. H. Tavabi, R. E. Dunin-Borkowski, C. Nobile, A. Cola, P. D. Cozzoli, S. Gambino and A. Rizzo, Sci. Rep., 2019, 9, $1-13$.

42 X. Ding, F. Peng, J. Zhou, W. Gong, G. Slaven, K. P. Loh, C. T. Lim and D. T. Leong, Nat. Commun., 2019, 10, 1-13.

43 V. Štengl and J. Henych, Nanoscale, 2013, 5, 3387-3394.

44 P. Sharma, A. Kumar, S. Bankuru, J. Chakraborty and S. Puravankara, New J. Chem., 2020, 44, 1594-1608.

45 N. Kumar, B. P. A. George, H. Abrahamse, V. Parashar and J. C. Ngila, Appl. Surf. Sci., 2017, 396, 8-18.

46 S. Masimukku, Y. C. Hu, Z. H. Lin, S. W. Chan, T. M. Chou and J. M. Wu, Nano Energy, 2018, 46, 338-346. 
47 M. B. Askari, A. F. Kalourazi, M. Seifi, S. S. Shahangian, N. Askari and T. J. Manjili, Optik, 2018, 174, 154-162.

48 J. Wang, N. Wang, Y. Guo, J. Yang, J. Wang, F. Wang, J. Sun, H. Xu, Z. H. Liu and R. Jiang, ACS Sustainable Chem. Eng., 2018, 6, 13435-13442.

49 J. Xie, H. Zhang, S. Li, R. Wang, X. Sun, M. Zhou, J. Zhou, X. W. Lou and Y. Xie, Adv. Mater., 2013, 25, 5807-5813.

50 H. Chu, X. Liu, B. Liu, G. Zhu, W. Lei, H. Du, J. Liu, J. Li, C. Li and C. Sun, Sci. Rep., 2016, 6, 1-10.

51 Y. Lu, X. Yao, J. Yin, G. Peng, P. Cui and X. Xu, RSC Adv., 2015, 5, 7938-7943.

52 F. Giubileo, A. Grillo, M. Passacantando, F. Urban, L. Iemmo, G. Luongo, A. Pelella, M. Loveridge, L. Lozzi and A. di Bartolomeo, Nanomaterials, 2019, 9, 717.

53 N. T. Thang, L. T. Hong, N. H. Thoan, C. M. Hung, N. van Duy, N. van Hieu and N. D. Hoa, RSC Adv., 2020, 10, 12759-12771.

54 Y. Sun, W. He, X. Sun and B. Liu, Luminescence, 2020, 1-8, DOI: $10.1002 /$ bio.3906.

55 Y. Sun, J. Xu, Z. Zhu, Y. Wang, H. Xia, Z. You, C. Lee and C. Tu, Opt. Mater. Express, 2015, 5, 2924.

56 Y. Wang, F. Zhang, Q. Wang, P. Yang, H. Lin and F. Qu, Nanoscale, 2018, 10, 14534-14545.

57 D. Son, S. I. Chae, M. Kim, M. K. Choi, J. Yang, K. Park, V. S. Kale, J. H. Koo, C. Choi, M. Lee, J. H. Kim, T. Hyeon and D.-H. Kim, Adv. Mater., 2016, 28, 9326-9332.

58 P. Schiettecatte, P. Geiregat and Z. Hens, J. Phys. Chem. C, 2019, 123, 10571-10577.

59 S. Barua, H. S. Dutta, S. Gogoi, R. Devi and R. Khan, ACS Appl. Nano Mater., 2018, 1, 2-25.

60 H. Laroui, D. S. Wilson, G. Dalmasso, K. Salaita, N. Murthy, S. v. Sitaraman and D. Merlin, Am. J. Physiol., 2011, 300, G371-G383.

61 V. Agarwal and K. Chatterjee, Nanoscale, 2018, 10, 16365-16397.

62 J. v. Jokerst, T. Lobovkina, R. N. Zare and S. S. Gambhir, Nanomedicine, 2011, 6, 715-728.

63 P. Milla, F. Dosio and L. Cattel, Curr. Drug Metab., 2012, 13, 105-119.

64 Z. Xie, Y. Duo, Z. Lin, T. Fan, C. Xing, L. Yu, R. Wang, M. Qiu, Y. Zhang, Y. Zhao, X. Yan and H. Zhang, Adv. Sci., 2020, 7, 1902236.

65 H. Chen, T. Liu, Z. Su, L. Shang and G. Wei, Nanoscale Horiz., 2018, 3, 74-89.

66 A. Doughty, A. Hoover, E. Layton, C. Murray, E. Howard and W. Chen, Materials, 2019, 12, 779.

67 C. Wang, J. Bai, Y. Liu, X. Jia and X. Jiang, ACS Biomater. Sci. Eng., 2016, 2, 2011-2017.

68 Z. Lei, W. Zhu, S. Xu, J. Ding, J. Wan and P. Wu, ACS Appl. Mater. Interfaces, 2016, 8, 20900-20908.

69 X. Yang, J. Li, T. Liang, C. Ma, Y. Zhang, H. Chen, N. Hanagata, H. Su and M. Xu, Nanoscale, 2014, 6, 10126-10133.

70 R. Parsons, Trans. Faraday Soc., 1958, 54, 1053-1063.

71 B. Hinnemann, P. G. Moses, J. Bonde, K. P. Jørgensen, J. H. Nielsen, S. Horch, I. Chorkendorff and J. K. Nørskov, J. Am. Chem. Soc., 2005, 127, 5308-5309.
72 A. B. Laursen, S. Kegnæs, S. Dahl and I. Chorkendorff, Energy Environ. Sci., 2012, 5, 5577-5591.

73 V. Nguyen, T. P. Nguyen, T. Le, D. N. Vo, D. L. Nguyen, Q. T. Trinh, I. T. Kim and Q. van Le, J. Chem. Technol. Biotechnol., 2020, 95, 2597-2607.

74 S. S. Chou, N. Sai, P. Lu, E. N. Coker, S. Liu, K. Artyushkova, T. S. Luk, B. Kaehr and C. J. Brinker, Nat. Commun., 2015, 6, 1-8.

75 S. Jayabal, J. Wu, J. Chen, D. Geng and X. Meng, Mater. Today Energy, 2018, 10, 264-279.

76 D. Voiry, A. Mohite and M. Chhowalla, Chem. Soc. Rev., 2015, 44, 2702-2712.

77 W. Zhou, X. Zou, S. Najmaei, Z. Liu, Y. Shi, J. Kong, J. Lou, P. M. Ajayan, B. I. Yakobson and J. C. Idrobo, Nano Lett., 2013, 13, 2615-2622.

78 N. García Doménech, F. Purcell-Milton and Y. K. Gun’ko, Mater. Today Commun., 2020, 23, 100888.

79 X. He and P. Li, Exposure Health, 2020, 12, 385-401.

80 S. Mishra and R. N. Bharagava, J. Environ. Sci. Health, Part C: Environ. Carcinog. Ecotoxicol. Rev., 2016, 34, 1-32.

81 M. Giagnorio, S. Steffenino, L. Meucci, M. C. Zanetti and A. Tiraferri, J. Environ. Chem. Eng., 2018, 6, 4467-4475.

82 G. Reina, J. M. González-Domínguez, A. Criado, E. Vázquez, A. Bianco and M. Prato, Chem. Soc. Rev., 2017, 46, 4400-4416.

83 S. Tang, W. Wu, X. Xie, X. Li and J. Gu, RSC Adv., 2017, 7, 9862-9871.

84 Z. Qian, L. Jiao and L. Xie, Chin. J. Chem., 2020, 38, 753-760.

85 A. A. Green and M. C. Hersam, J. Phys. Chem. Lett., 2010, 1, 544-549.

86 M. G. Stanford, P. D. Rack and D. Jariwala, npj 2D Mater. Appl., 2018, 2, 20.

87 S. Jeong, D. Yoo, M. Ahn, P. Miro, T. Heine and J. Cheon, Nat. Commun., 2015, 6, 1-7.

$88 \mathrm{~J}$. Li, M. C. Lemme and M. Östling, ChemPhysChem, 2014, 15, 3427-3434.

89 J. Wang and B. Liu, Sci. Technol. Adv. Mater., 2019, 20, 992-1009.

90 D. Ovchinnikov, A. Allain, Y. S. Huang, D. Dumcenco and A. Kis, ACS Nano, 2014, 8, 8174-8181.

91 R. H. Fowler and L. Nordheim, Proc. R. Soc. London, Ser. A, 1928, 119, 173-181.

92 F. Giubileo, A. di Bartolomeo, L. Iemmo, G. Luongo and F. Urban, Appl. Sci., 2018, 8, 526.

93 F. Urban, M. Passacantando, F. Giubileo, L. Iemmo and A. di Bartolomeo, Nanomaterials, 2018, 8(3), 151.

94 W. B. Choi, D. S. Chung, J. H. Kang, H. Y. Kim, Y. W. Jin, I. T. Han, Y. H. Lee, J. E. Jung, N. S. Lee, G. S. Park and J. M. Kim, Appl. Phys. Lett., 1999, 75, 3129-3131.

95 R. Yabushita, K. Hata, H. Sato and Y. Saito, J. Vac. Sci. Technol., B: Microelectron. Nanometer Struct.-Process., Meas., Phenom., 2007, 25, 640.

96 T. Cao, G. Wang, W. Han, H. Ye, C. Zhu, J. Shi, Q. Niu, P. Tan, E. Wang, B. Liu and J. Feng, Nat. Commun., 2012, 3, 1-5.

97 K. F. Mak, K. He, J. Shan and T. F. Heinz, Nat. Nanotechnol., 2012, 7, 494-498. 
98 W. Yao, D. Xiao and Q. Niu, Phys. Rev. B: Condens. Matter Mater. Phys., 2008, 77, 235406.

99 F. Purcell-Milton, R. McKenna, L. J. Brennan, C. P. Cullen, L. Guillemeney, N. v. Tepliakov, A. S. Baimuratov, I. D. Rukhlenko, T. S. Perova, G. S. Duesberg, A. v. Baranov, A. v. Fedorov and Y. K. Gun'Ko, ACS Nano, 2018, 12, 954-964. 100 V. Galstyan, Sensors, 2017, 17, 2947.

101 A. J. Bandodkar, I. Jeerapan and J. Wang, ACS Sens., 2016, 1, 464-482.

102 M. C. Domingo, J. Network Comput. Appl., 2012, 35, 584-596. 103 W. Yang, L. Gan, H. Li and T. Zhai, Inorg. Chem. Front., 2016, 3, 433-451.

104 D. J. Late, Y. K. Huang, B. Liu, J. Acharya, S. N. Shirodkar, J. Luo, A. Yan, D. Charles, U. v. Waghmare, V. P. Dravid and C. N. R. Rao, ACS Nano, 2013, 7, 4879-4891.

105 W. Yin, X. Dong, J. Yu, J. Pan, Z. Yao, Z. Gu and Y. Zhao, ACS Appl. Mater. Interfaces, 2017, 9, 21362-21370.

106 D. Qiu, Y. Chu, H. Zeng, H. Xu and G. Dan, ACS Appl. Mater. Interfaces, 2019, 11, 37035-37042.

107 A. Ganeshpurkar and A. K. Saluja, Saudi Pharm. J., 2017, 25, 149-164.

108 F. Yi, H. Ren, J. Shan, X. Sun, D. Wei and Z. Liu, Chem. Soc. Rev., 2018, 47, 3152-3188.

109 J. R. Sootsman, D. Y. Chung and M. G. Kanatzidis, Angew. Chem., Int. Ed., 2009, 48, 8616-8639.

110 Y. Xie, T. M. Chou, W. Yang, M. He, Y. Zhao, N. Li and Z. H. Lin, Semicond. Sci. Technol., 2017, 32, 044003.

111 D. D. Fan, H. J. Liu, L. Cheng, P. H. Jiang, J. Shi and X. F. Tang, Appl. Phys. Lett., 2014, 105, 133113.

112 C. Shang, Y. Zhang, H. Qin, B. He, C. Zhang, J. Sun, J. Li, J. Ma, X. Ji, L. Xu and B. Fu, Opt. Laser Technol., 2020, 131, 106375.

113 Z. Luo, D. Wu, B. Xu, H. Xu, Z. Cai, J. Peng, J. Weng, S. Xu, C. Zhu, F. Wang, Z. Sun and H. Zhang, Nanoscale, 2016, 8, 1066-1072.

114 A. Martinez and Z. Sun, Nat. Photonics, 2013, 7, 842-845.
115 W. Jie, Z. Yang, G. Bai and J. Hao, Adv. Opt. Mater., 2018, 6, 1701296.

116 W. Zhao, Z. Ghorannevis, L. Chu, M. Toh, C. Kloc, P. H. Tan and G. Eda, ACS Nano, 2013, 7, 791-797.

117 S. Mouri, Y. Miyauchi and K. Matsuda, Nano Lett., 2013, 13, 5944-5948.

118 M. Amani, D. H. Lien, D. Kiriya, J. Xiao, A. Azcatl, J. Noh, S. R. Madhvapathy, R. Addou, K. C. Santosh, M. Dubey, K. Cho, R. M. Wallace, S. C. Lee, J. H. He, J. W. Ager, X. Zhang, E. Yablonovitch and A. Javey, Science, 2015, 350, 1065-1068.

119 A. Pospischil, M. M. Furchi and T. Mueller, Nat. Nanotechnol., 2014, 9, 257-261.

120 J. L. O’Brien, A. Furusawa and J. Vučković, Nat. Photonics, 2009, 3, 687-695.

121 Y. J. Zhang, T. Oka, R. Suzuki, J. T. Ye and Y. Iwasa, Science, 2014, 344, 725-728.

122 G. Liu, W. Jin and N. Xu, Angew. Chem., Int. Ed., 2016, 55, 13384-13397.

123 L. Sun, Y. Ying, H. Huang, Z. Song, Y. Mao, Z. Xu and X. Peng, ACS Nano, 2014, 8, 6304-6311.

124 L. Sun, H. Huang and X. Peng, Chem. Commun., 2013, 49, 10718-10720.

125 M. Samadi, N. Sarikhani, M. Zirak, H. Zhang, H. L. Zhang and A. Z. Moshfegh, Nanoscale Horiz., 2018, 3, 90-204.

126 Y. Abate, D. Akinwande, S. Gamage, H. Wang, M. Snure, N. Poudel and S. B. Cronin, Adv. Mater., 2018, 30, 1704749.

127 R. Ma and T. Sasaki, Acc. Chem. Res., 2015, 48, 136-143.

128 G. Liu, W. Jin and N. Xu, Angew. Chem., Int. Ed., 2016, 55, 13384-13397.

129 K. S. Novoselov, A. Mishchenko, A. Carvalho and A. H. Castro Neto, Science, 2016, 353.

130 E. H. Ang and J. W. Chew, Chem. Mater., 2019, 31, 10002-10007.

131 H. M. Tham, S. Japip and T. S. Chung, J. Membr. Sci., 2019, 588, 117219. 